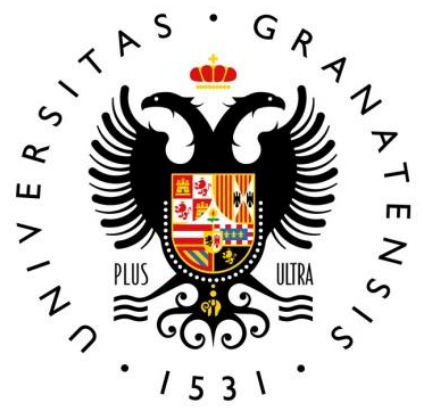

UNIVERSIDAD DE GRANADA

FACULTAD DE CIENCIAS DE LA EDUCACIÓN

DEPARTAMENTO DE DIDÁCTICA DE LA MATEMÁTICA

\title{
PENSAMIENTO FUNCIONAL CON VARIABLES CONTINUAS EN ALUMNOS DE EDUCACIÓN PRIMARIA EN UN PROGRAMA DE ENRIQUECIMIENTO CURRICULAR
}

Presentado por

Dña. Alba María Damián Gómez

Dirigido por

Dña. María C. Cañadas Santiago y D. Rafael Ramírez Uclés

Granada, 2020 


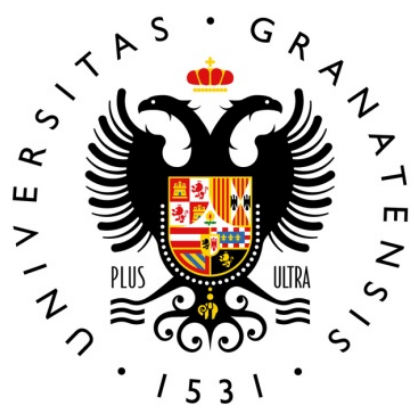

\title{
PENSAMIENTO FUNCIONAL CON VARIABLES CONTINUAS EN ALUMNOS DE EDUCACIÓN PRIMARIA DE UN PROGRAMA DE ENRIQUECIMIENTO CURRICULAR
}

\author{
Trabajo de Fin de Máster presentado por \\ Dña. Alba María Damián Gómez \\ para la obtención del título Máster en Didáctica de la Matemática
}

Dña. Alba María Damián Gómez

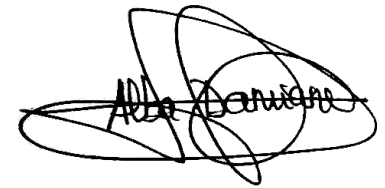

Tutores

Dña. María C. Cañadas Santiago

D. Rafael Ramírez Uclés
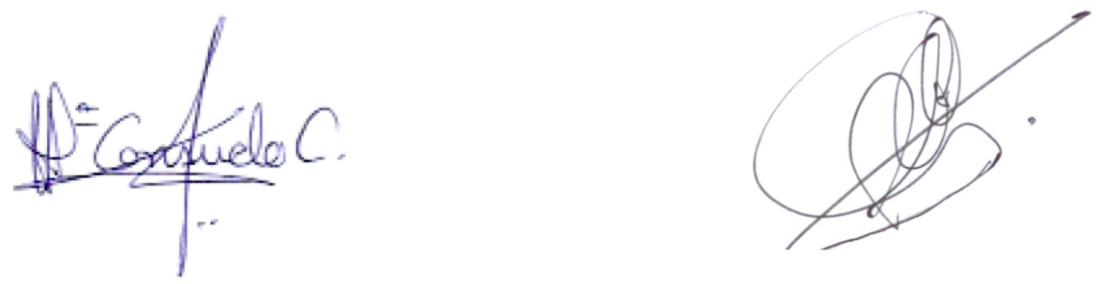

Granada, 2020 
El presente trabajo de investigación se ha realizado dentro del proyecto de investigación del Plan Nacional I+D con referencia EDU2016-75771-P, financiado por el Ministerio de Economía y Competitividad de España; y en el seno del grupo de investigación FQM193: "Didáctica de la Matemática. Pensamiento Numérico" (FQM-193) de la Universidad de Granada, perteneciente al Plan Andaluz de Investigación, Desarrollo e Innovación de la Junta de Andalucía. 


\section{Resumen}

Presentamos una investigación cuyo objetivo es describir el pensamiento funcional de seis alumnos de $6^{\circ}$ de primaria que pertenecen a un programa de enriquecimiento curricular. Les proponemos una tarea de generalización que involucra una función cuadrática de variable continua. En cuanto al tipo de valores que puede tomar la variable independiente, algunos estudiantes asumieron que la variable puede tomar valores decimales, pero no negativos. Hay estudiantes que identificaron la estructura $L \times L$, aunque no lo expresaron como potencia. Los alumnos usan varias representaciones, en su mayoría verbal y simbólica, aunque también aparecen representaciones pictóricas y múltiples.

\section{Summary}

We present a study whose objective is to describe the functional thinking of six graders who belong to a curriculum enrichment program. We propose a generalization task that involves a quadratic function of continuous variable. Concerning the kind of values of the independent variable, some students assumed that it can be a decimal number, but not negative number. There students who identified the structure $L \times L$, although they did not express as a potential expression. The students used various representations, mostly verbal and symbolic, although pictorial and multiple representations also appear. 


\section{Índice}

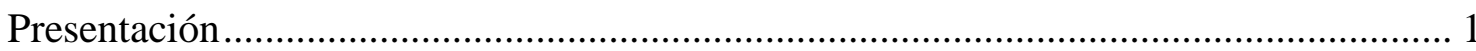

Capítulo 1. Presentación y justificación del problema de investigación ........................... 3

1.1. Presentación del problema de investigación ..................................................... 3

1.2. Justificación del problema de investigación ..................................................... 4

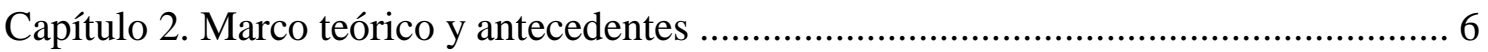



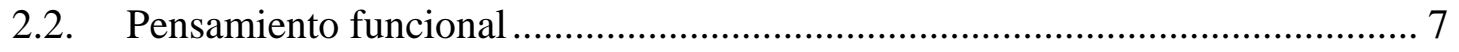

2.3. La función como foco matemático del pensamiento funcional ........................ 8

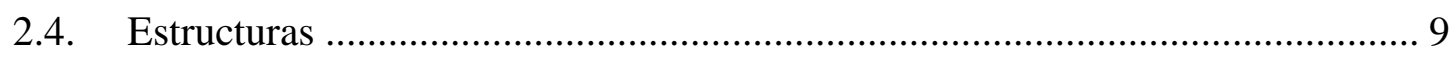

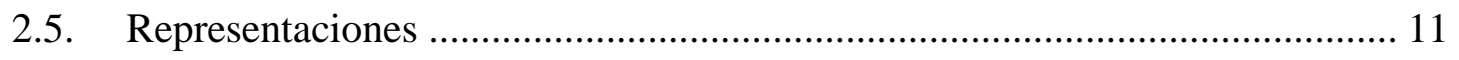

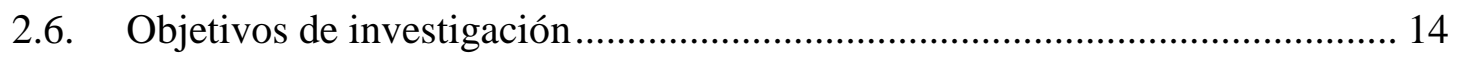



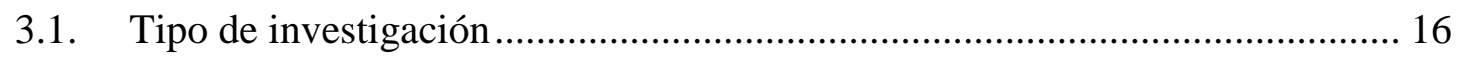



3.3. Instrumento de recogida de información ....................................................... 19











4.2. Puesta en común ....................................................................................... 31

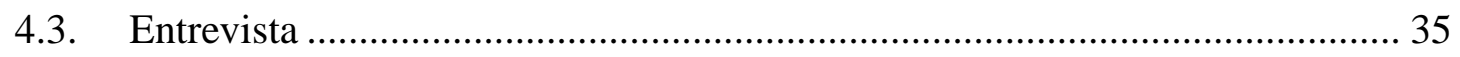



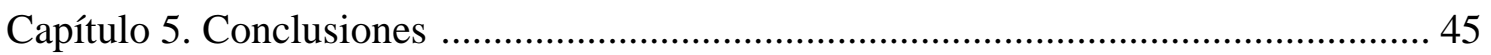

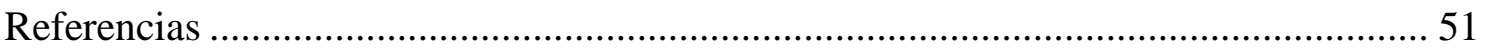




\section{Índice de tablas}

Tabla 1. Valores extremos en el trabajo en el cuestionario .......................................... 28

Tabla 2. Valores en el trabajo en el cuestionario......................................................... 29

Tabla 3. Estructuras y representación en el trabajo en el cuestionario ......................... 30

Tabla 4. Valores extremos en la puesta en común ........................................................ 31

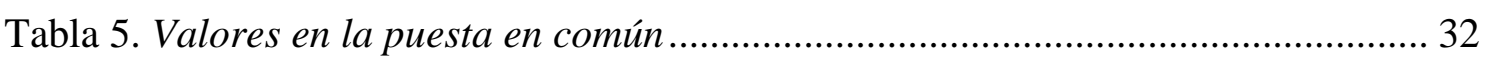

Tabla 6. Representación en la puesta en común .............................................................. 35

Tabla 7. Valores extremos en la tarea de las 4 esquinas en la entrevista....................... 36

Tabla 8. Valores extremos en la tarea de las 2 esquinas en la entrevista...................... 37

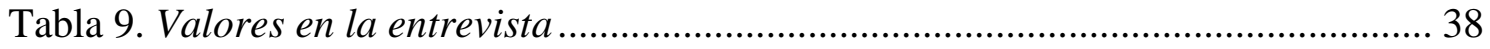

Tabla 10. Estructuras en la tarea de las 4 esquinas en la entrevista ............................. 39

Tabla 11. Estructuras en la tarea de las 2 esquinas en la entrevista ............................ 40



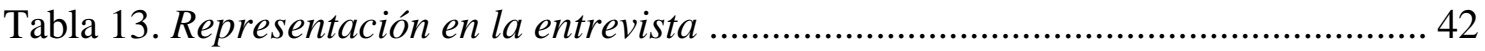

\section{Índice de figuras}



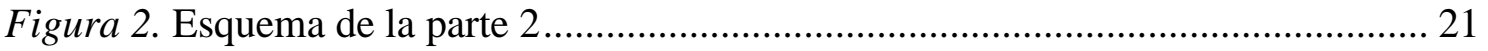





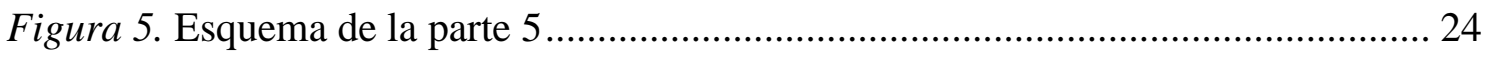

Figura 6. Desarrollo plano usado para generar cajas ................................................ 25

Figura 7. Representaciones pictórica y simbólica usadas por los investigadores.......... 34 


\section{Presentación}

El presente documento corresponde al Trabajo Fin de Máster (TFM) realizado por la alumna Alba María Damián Gómez, dentro del programa de Máster en Didáctica de la Matemática de la Universidad de Granada, durante el curso académico 2019/2020 y dirigido por María C. Cañadas Santiago y Rafael Ramírez Uclés.

El foco principal de este TFM es el pensamiento funcional de estudiantes que participan en un programa de enriquecimiento curricular. La motivación para la elección de dicho foco reside en el interés manifiesto del pensamiento funcional en los primeros niveles educativos y la inexistencia de investigaciones en el colectivo mencionado. Si bien existen estudios relacionados con el pensamiento funcional (e.g., Cañadas y Fuentes, 2015; Castro, Cañadas y Molina, 2017) y los programas de enriquecimiento curricular (e.g., Benavides, Maz, Castro y Blanco, 2004), no se encuentran en la literatura documentos que los conecten.

En este estudio trabajamos con alumnos de $6^{\circ}$ de educación primaria que pertenecen a un programa de enriquecimiento curricular mediante el uso de una tarea que implica una función cuadrática de variable continua. Esto último supone una novedad en el presente trabajo, dado que no se corresponde con el tipo de funciones que usualmente se proponen en investigaciones sobre pensamiento funcional en educación primaria, donde las funciones suelen ser lineales y los valores discretos.

Este informe de investigación se compone de seis capítulos. En el primero de ellos planteamos el problema de investigación y su justificación en los ámbitos curricular e investigativo.

El segundo capítulo contiene el marco conceptual en que se apoya la investigación y los antecedentes existentes sobre el tema objeto de estudio. Con base en estos elementos, el tercer capítulo expone los objetivos generales y específicos de la investigación.

En el cuarto capítulo describimos la metodología utilizada en el estudio. Exponemos la naturaleza y el enfoque de la investigación, los sujetos, el método utilizado para la recogida de datos y las categorías y el método de análisis. 
En el quinto capítulo presentamos el análisis de los datos y los resultados obtenidos con base en los objetivos de investigación fijados. Por último, en el sexto capítulo describimos las conclusiones de la investigación relativas a la consecución de los objetivos, los aportes del estudio, las limitaciones encontradas en el proceso y las líneas de investigación que quedan abiertas. 


\section{Capítulo 1. Presentación y justificación del problema de investigación}

En este capítulo presentamos y justificamos el interés del problema de investigación.

\subsection{Presentación del problema de investigación}

Desde la década de los 90 se han llevado a cabo múltiples investigaciones en torno al desarrollo del pensamiento algebraico en educación primaria. Con las primeras investigaciones surge la conocida propuesta curricular conocida como early algebra, que busca promover modos de pensamiento algebraico, generar un mayor grado de generalización en el pensamiento de alumnos de educación primaria y aumentar su capacidad para expresar la generalización (e.g., Brizuela y Blanton, 2014; Molina, 2005). Todo esto desde la "algebrización del currículum" (Kaput, 2000).

Uno de los focos de investigación en el contexto de la propuesta early algebra es el desarrollo del pensamiento funcional en estudiantes. En la última década se han publicado diferentes estudios en este contexto con estudiantes en España (e.g., Ayala-Altamirano y Molina, 2019; Morales, Cañadas, Brizuela y Gómez, 2018; Pinto y Cañadas, 2019; Pinto, Cañadas, Moreno y Castro, 2016; Ureña, Ramírez y Molina, 2019). Estos autores indagan en las relaciones funcionales y las representaciones de las mismas usadas por los estudiantes, entre otros aspectos. Hasta ahora las investigaciones se han centrado en el trabajo con funciones de variable discreta. Consideramos interesante explorar también las variables continuas, pues describen muchos de los fenómenos que podrían contextualizar otros problemas con funciones.

Nuestro interés es indagar en el pensamiento funcional de los estudiantes cuando la tarea implicada contiene una función de variable continua. Dada la mayor complejidad de estas variables, vamos a indagar inicialmente en estudiantes que forman parte de un programa de enriquecimiento curricular, habiendo sido nominados como talento matemático por sus tutores. El interés en dicho colectivo es minimizar las potenciales dificultades que podrían derivarse de la falta de conocimientos matemáticos previos o de una actitud negativa hacia las tareas matemáticas propuestas. 


\subsection{Justificación del problema de investigación}

Justificamos el problema de investigación con base en dos dimensiones: curricular e investigadora.

\section{Justificación curricular}

La incorporación del álgebra y de los elementos relacionados con el pensamiento funcional en educación primaria no ha estado ni está presente en los diseños curriculares de la mayoría de los países. Estados Unidos es el primer país que presenta un documento que incluye el álgebra en el currículo de educación primaria: Principles and standards for school mathematics (NCTM, 2000). Este documento explicita que los estudiantes necesitan "comprender sus conceptos (del álgebra), las estructuras y los principios que rigen la manipulación de los símbolos, y cómo pueden usarse éstos para registrar ideas y ampliar su comprensión de las situaciones" (p. 39). Sin embargo, nueve años más tarde, los líderes estatales promueven, a través de la National Governors Association Center for Best Practices (NGA Center) y el Council of Chief State School Officers (CCSSO), los Common Core como estándares comunes, rigiéndose la mayor parte de Estados Unidos por ellos en la actualidad. Estos estándares introducen durante toda la etapa de educación primaria nociones como número desconocido, relación entre cantidades, relación entre operaciones, patrones o secuencias (NGA y CCSSO, 2010). Otros países, como Australia, Canadá, China, Corea, Japón o Portugal, también han recogidos ideas relacionadas con la inclusión de nociones algebraicas en los primeros cursos de educación primaria (Merino, Cañadas y Molina, 2013).

En España, país en el que se desarrolla este trabajo, el último currículo básico de educación primaria se encuentra recogido en el Real Decreto 126/2014 (MECD, 2014). En el bloque 1, relativo a procesos, métodos y actitudes en matemáticas, bloque transversal en la materia de matemáticas, aparece un elemento relativo al pensamiento funcional. En dicho bloque aparece lo que se espera de los estudiantes al terminar esta etapa educativa, buscando que estos sean capaces de "describir y analizar situaciones de cambio, encontrar patrones, regularidades y leyes matemáticas en contextos numéricos, geométricos y funcionales, valorando su utilidad para hacer predicciones" (p. 19.387).

En referencia al uso de la variable continua y cuadrática en contextos geométricos en nuestro trabajo, encontramos que el Real Decreto 126/2014 establece en el bloque 2, relativo al uso de los números, como contenidos curriculares los números decimales y las 
fracciones, la potencia como producto de factores iguales, los cuadrados y cubos y las operaciones con fracciones y números decimales; en el bloque 3 , relativo a la medida, la comparación de superficies de figuras planas por superposición, descomposición y medición y la estimación de superficies de objetos y espacios conocidos; en el bloque 4, relativo a geometría, las áreas de figuras planas.

\section{Justificación investigadora}

El presente trabajo se enmarca dentro de la propuesta curricular early algebra. En concreto, nos focalizamos en la concepción del álgebra escolar como estudio de las funciones y en el pensamiento funcional de los estudiantes de educación primaria. Existen evidencias de la importancia de promover este tipo de pensamiento en los estudiantes de los primeros niveles educativos (Cañadas y Fuentes, 2015; Torres, Cañadas y Moreno, 2019). Dentro de dicho contexto, varias investigaciones se interesan por las estructuras que identifican y las representaciones que emplean los estudiantes. Estos son elementos de interés para nuestro estudio. Destacamos, entre otros, los trabajos de Merino et al. (2013), Cañadas y Fuentes (2015), Pinto y Cañadas (2019) y Ureña et al. (2019) en el contexto nacional. En el contexto internacional, están Wilkie (2016), Blanton y Kaput (2004) o Blanton, Gardiner, Sawrey y Newman-Owens (2015), entre otros. Todos ellos contienen como elemento común el tipo de variables y el tipo de función involucrados - funciones lineales de variables discretas. Este hecho, junto con la falta de estudios e investigaciones en la literatura que impliquen variables continuas o funciones de orden superior a uno sugieren la necesidad de indagar en el pensamiento funcional de los estudiantes.

Planteamos el estudio en un contexto particular y distinto al usual: los programas de enriquecimiento curricular. Buscamos con ello que las potenciales dificultades matemáticas queden discriminadas por el hecho de ser alumnos con buena actitud hacia las tareas matemáticas y presencia de conocimientos previos afianzados. 


\section{Capítulo 2. Marco teórico y antecedentes}

El marco teórico tiene como foco principal el pensamiento funcional. Ubicamos este proceso cognitivo en un contexto más general: el álgebra escolar. A continuación, describimos la noción matemática protagonista - la función- y algunos de sus elementos, particularmente en los que nos centramos en esta investigación: estructuras y representaciones. En cada una de las secciones incorporamos ideas relativas del marco teórico y los principales antecedentes de nuestro trabajo.

\subsection{Enfoque funcional del álgebra escolar}

El álgebra escolar hace referencia al álgebra que se enseña en la enseñanza básica (de 6 a 12 años). Si bien parece un concepto sencillo en su definición, muchos son los autores, como por ejemplo Kaput (1999), Usiskin (1988) y Kaput, Carraher y Blanton (2008), que han tratado de definir lo que ellos mismos conciben como álgebra escolar o lo que se entiende de forma general en la comunidad investigadora en Educación Matemática. Es en el proceso de definición donde surgen las diferentes aproximaciones, generándose así una visión multidisciplinar del álgebra escolar. Los enfoques del álgebra escolar que Molina (2015) identifica son: (a) aritmética generalizada y estudio de patrones, (b) funciones, (c) resolución de problemas, (d) estudio de estructuras y (e) lenguaje algebraico. Estas concepciones no son disjuntas entre sí dado que están relacionadas y su práctica en el aula no es separada (Drijvers, 2003). No es posible pensar en el álgebra solamente como parte de una de estas concepciones. Kaput et al. (2008) exponen que considerar las concepciones del álgebra como departamentos separados resulta un obstáculo al tratar de identificar modos en que el aprendizaje puede partir de las habilidades y conocimientos previos de los estudiantes. En este trabajo ponemos nuestro énfasis en la concepción del álgebra como estudio de las funciones. Esta concepción es el estudio de las relaciones entre las variables aplicadas a situaciones reales explicadas mediante modelos. Varias razones justifican nuestro interés por el enfoque funcional desde los primeros cursos de educación primaria.

- Supone una ayuda para superar las dificultades existentes en la comprensión del concepto de función en educación secundaria (Doorman y Drijvers, 2011).

- Fomenta la capacidad para generalizar, representar, justificar y razonar con relaciones matemáticas (Blanton, Levi, Crites y Dougherty, 2011). 
- El trabajo con funciones favorece la comprensión de variables, la manipulación de fórmulas y el paso entre diferentes representaciones.

Diferentes autores hacen mención a la introducción de las funciones en la educación primaria. Según Usiskin (1988), es relevante el papel de las funciones, así como el tiempo de introducción a los alumnos. El autor sostiene que algunos currículos han argumentado que las funciones pueden ser usadas como el principal vehículo con el que las variables y el álgebra son introducidos, si bien critica que se trata como tema insignificante en los libros de primer curso, dándole mayor importancia en los libros de cursos posteriores. Autores como Drijvers, Dekker y Wijers (2011) coinciden con esto y recomiendan el enfoque funcional, incluso en los primeros cursos de Educación Primaria, dado el uso del álgebra en situaciones concretas de una forma significativa que ello supone.

Para Heid (1996), el enfoque funcional del álgebra es un estudio centrado en el desarrollo de experiencias con funciones y familias de funciones en situaciones de la vida real en las que las relaciones cuantitativas puedan explicarse por medio de modelos.

La literatura expone que los estudiantes de educación primaria pueden llegar a describir relaciones recursivas, covariación y correspondencia entre cantidades, haciendo uso de diferentes sistemas de representación para ello (Moss, Beatty, Shillolo y Barkin, 2008) así como identificar propiedades generales a partir de situaciones particulares cuando existe una relación entre dos cantidades que cambian (Mason, Stephens y Watson, 2009; Warren y Cooper, 2005).

\subsection{Pensamiento funcional}

Cuando el foco matemático del pensamiento algebraico es la función, se habla de pensamiento funcional (Cañadas, Brizuela y Blanton, 2016). Según Smith (2008), el pensamiento funcional es una actividad cognitiva de las personas que se centra en la relación entre dos o más cantidades que varían. Destaca también la implicación que tiene el trabajo con las relaciones funcionales para avanzar en la construcción de ideas clave acerca de la noción de función. Es una meta disciplinar y esencial en la enseñanza de las matemáticas, aquella que se refiere a la acción de "pensar en términos y acerca de relaciones" (Rico, 2007, p. 56). 
Se denomina pensamiento funcional al proceso de construir, describir y razonar con y sobre funciones, entendiendo como función la relación de dependencia entre cantidades covariantes. Es dicha función el foco de contenido matemático en el que se centra el pensamiento funcional (Cañadas, 2016). "Es una componente del pensamiento algebraico basada en la construcción, descripción, representación y razonamiento con y sobre las funciones y los elementos que las constituyen” (Cañadas y Molina, 2016, p. 3).

Las cantidades variables, sus relaciones, la recursividad, la correspondencia entre valores de las variables o el uso de diferentes sistemas de representación en un contexto de resolución de problemas son algunos de los elementos clave necesarios para el desarrollo del pensamiento funcional desde los primeros cursos (Blanton et al., 2011). Estos autores exponen como características del pensamiento funcional: (a) la generalización de relaciones entre cantidades covariantes, (b) la representación de dichas relaciones de diferentes formas haciendo uso del lenguaje natural, (c) las expresiones simbólicas, las tablas y los gráficos y (d) el razonamiento de manera fluida con esas representaciones para interpretar y predecir el comportamiento de las funciones.

\subsection{La función como foco matemático del pensamiento funcional}

El foco de contenido matemático principal del pensamiento funcional es la función. Originalmente, la noción de función nace para permitir el estudio de relaciones existentes entre cantidades cambiantes en el mundo físico (Lloyd, Beckmann, Zbiek y Cooney, 2010). En la literatura, son varias las definiciones que se encuentran para este objeto matemático. Se entiende por función "la ley que asigna a cada elemento de un conjunto A un único elemento de un conjunto B (donde B puede o no ser igual a A)" (Usiskin, Peressino, Marchisotto y Stanley, 2003, p. 68).

Blanton et al. (2011) establecen como función "la relación especial entre dos conjuntos, en la cual cada elemento de un conjunto, llamado dominio, está asociado únicamente a un elemento del segundo conjunto, llamado codomino" (p. 48). En una función, los valores de una variable varían según los valores de la otra, siendo la primera la variable dependiente y la segunda, la independiente. Freudenthal (1983) expone la importancia fenomenológica de la función como relación entre algo que varía libremente y algo que varía bajo determinadas limitaciones (p. 496).

La variable de la función usada en el presente documento es una variable continua. Se entiende por variable continua aquella cuyos posibles valores son todos los valores 
pertenecientes a un intervalos, y por tanto, infinitos valores (Asencio, Romero y de Vicente, 2003).

En cuanto al trabajo de la función en el aula, Confrey y Smith (1991) consideran la resolución de problemas contextualizados para la enseñanza de las funciones, involucrando representaciones múltiples y transformaciones de las mismas.

Sin embargo, la función no es un contenido curricular de los primeros cursos, por lo que existen muchas cuestiones abiertas sobre cómo abordar el pensamiento funcional con los niños de dichos niveles educativos (Schliemann, Carraher y Brizuela, 2012). La atención que se da al desarrollo del pensamiento funcional en los primeros niveles educativos a pesar de la importancia del mismo es escasa, surgiendo así un problema de investigación (Blanton y Kaput, 2011). Hasta hace menos de una década, el estudio de este contenido curricular estaba relegado a la etapa de Educación Secundaria. Cañadas y Molina (2016) exponen acerca del pensamiento funcional:

No se trata de introducir las funciones en niveles educativos previos tal y como se trabajan en educación secundaria, sino de aprovechar el potencial de este contenido matemático para promover capacidades en los niños que les sean útiles para el razonamiento en general y el matemático en particular, tanto en el nivel educativo en el que se encuentran como en los sucesivos (p. 2).

Molina y Cañadas (2018) exponen que "la forma en que se trabajan las relaciones funcionales en el marco de la propuesta early algebra se basa en promover la percepción y generalización de patrones detectados en situaciones donde hay dos variables relacionadas que covarían" (p. 5). Se trabaja en situaciones en las que existen relaciones funcionales, estableciendo los alumnos variedad de estructuras de las mismas, no siendo estas en ocasiones adecuadas o equivalentes.

\subsection{Estructuras}

Se entiende por estructura matemática una vía en la que varios elementos, matemáticos en el presente contexto, se relacionan y organizan (Mulligan, English, Mitchelmore y Roberston, 2010), permitiendo el establecimiento de conexiones y relaciones ente 
conceptos y procesos matemáticos y, en consecuencia, el análisis de cómo los estudiantes interpretan y generalizan una regularidad (Warren, Miller y Cooper, 2013).

En el marco del early algebra, las regularidades en la visión funcional guardan relación con las relaciones entre las variables dependiente e independiente implicadas en la situación. La noción de estructura tiene diferentes concepciones en el marco del pensamiento algebraico, por su estrecha relación con la generalización (Molina y Cañadas, 2018). Mason et al. (2009) exponen que previamente a la generalización, los estudiantes deben ver la estructura. Algunos estudios demuestran que cuando identifican estructuras en una tarea matemática, los alumnos experimentan las matemáticas de una forma más profunda. La estructura se corresponde con el modo en el que se organizan los elementos de una regularidad y la relación existente entre ellos (Kieran, 1989). En la práctica, los estudiantes identifican variedad de estructuras, no teniendo que ser todas ellas correctas ni equivalentes (Molina y Cañadas, 2018).

En el seno del proyecto en el que se enmarca este trabajo, la noción de estructura la asociamos a la organización de la relación entre las variables percibida por los estudiantes. Desde la perspectiva del pensamiento funcional en la que se enmarca el presente trabajo, consideramos la noción de estructura como los números y las variables numéricas (expresadas mediante el uso de diferentes representaciones), operaciones y sus propiedades presentes (Pinto y Cañadas, 2018). Es decir, la estructura en el contexto funcional es equivalente a la regularidad.

Existen líneas de investigación en aspectos relacionados con las estructuras que los estudiantes utilizan en sus tareas. Por ejemplo, Torres et al. (2019) indagan en el pensamiento funcional de tres estudiantes de $2^{\circ}$ de educación primaria (7-8 años), mediante el uso de tareas que implican tres funciones lineales $f(x)=x+3$ (aditiva), la operación multiplicación $f(x)=2 x$ (multiplicativa) y la combinación de ambas $f(x)=$ $1+2 x$ (combinación de aditiva y multiplicativa). Como resultados principales, los autores evidencian la presencia de pocas estructuras diferentes por parte de los alumnos para una misma regularidad y la dificultad que presentan los mismos en el establecimiento de estructuras que implican una combinación de operaciones.

Pinto y Cañadas (2018) realizan un estudio a 24 estudiantes de $5^{\circ}$ de educación primaria (10-11 años) que implica la función lineal $f(x)=2 x+6$ (teniendo en cuenta las operaciones de adición y multiplicación). El artículo, que se corresponde con la cuarta 
sesión de un experimento de enseñanza más amplio, analiza el establecimiento de la relación funcional por parte de los alumnos y el tipo de representación usada para ello, sabiendo que los alumnos no habían trabajado previo al estudio con problemas que implicasen funciones. Se concluye que los alumnos son capaces de establecer la generalización y que 10 estudiantes establecen diferentes estructuras, expresadas de varias formas (hecho que permite interpretar y entender el proceso seguido por los estudiantes, según los autores). Se evidencia también que quizá algunos de los estudiantes son capaces de generalizar pero no lo expresan hasta que se les requiere.

Pinto y Cañadas (2019), realizan un estudio de nuevo con esta tarea con alumnos de $3^{\circ}$ (8-9 años) y $5^{\circ}$ de educación primaria, destacando de nuevo la amplia variedad de estructuras, que es mayor en $3^{\circ}$ que en $5^{\circ}$. Los autores evidencian un mayor número de estudiantes en $5^{\circ}$ (19 de 24$)$ que en $3^{\circ}$ (11 de 24) que establecen la estructura, asumiendo ello en el hecho de que los estudiantes de cursos superiores tienden a focalizar más en la relación entre cantidades. De hecho, los estudiantes de $3^{\circ}$ hacen uso de casos particulares, de valores específicos en sus producciones, demostrando así que no tienen la necesidad de encontrar la relación.

\subsection{Representaciones}

Rico (2009) subraya que la representación se basa en la dupla representante-representado e identifica las representaciones como "todas aquellas herramientas — signos o gráficos que hacen presentes los conceptos y procedimientos matemáticos y con las cuales los sujetos particulares abordan e interactúan con el conocimiento matemático, es decir, registran y comunican su conocimiento sobre las matemáticas” (p. 3). El interés didáctico de las diferentes representaciones que pueden establecerse estriba en el hecho de que mediante el trabajo con ellas, las personas asignan significados y comprenden las estructuras matemáticas antes mencionadas (Radford, 1998).

Una rica comprensión de las funciones se caracteriza por la fluidez representativa, esto es, la capacidad de representar funciones de múltiples maneras y navegar de manera flexible entre estas diversas representaciones (Blanton et al., 2011).

Un pensamiento matemático efectivo supone la comprensión de las relaciones entre las diferentes representaciones del mismo concepto, así como las semejanzas y diferencias 
estructurales entre ellas (Goldin y Shteingold, 2001). Rico (2009) expone que una representación única y aislada carece de sentido. Es por ello que debe contemplarse dentro de un sistema de significados y relaciones, por lo que resulta interesante definir y trabajar con sistemas de representación. Una de las características de los conceptos y estructuras matemáticas reside en la necesidad del empleo de diversas y diferentes representaciones para captarlos en toda su complejidad.

El uso de la representación verbal, numérica, gráfica o algebraica supone un extra en hacer el proceso de aprendizaje del álgebra significativo y efectivo, teniendo en cuenta cada una de las ventajas y desventajas que supone cada representación. Sin embargo, el uso de ellas en conjunto elimina de forma considerable las desventajas (Friedlander y Tabach, 2001). Estos autores exponen que la elección de una representación puede ser el resultado de la naturaleza de la tarea, la preferencia personal, el estilo de pensamiento del solucionador de problemas o los intentos de superar las dificultades encontradas durante el uso de otra representación. Con frecuencia, la elección de la representación está influenciada por una combinación de varios factores. Para responder una pregunta, los estudiantes pueden elegir una representación sobre la base de su análisis del problema y la preferencia personal, y pueden cambiar a otra representación en una etapa posterior como resultado de dificultades en el proceso de solución.

En general, los alumnos estarán en mejores condiciones para resolver una variedad de problemas de álgebra si pueden transformar fácilmente un tipo de representación en otro, considerando que la facilidad en la transformación puede surgir a medida que se adquiera la experiencia con múltiples formas de representar un problema contextualizado (NCTM, 2000, p. 281).

Existen diferentes clasificaciones de tipos de representaciones. Tomamos como referencia aquellos que Merino (2012) usó en su análisis de las producciones de los estudiantes en un contexto de pensamiento funcional. Para ello, el autor partió de la clasificación establecida por Kolloffel, Eysink, De Jong y Wilhelm (2009) para otros contenidos matemáticos, más tarde restructurada por Cañadas y Figueiras (2011). Merino (2012) identifica cinco sistemas de representación en su estudio: (a) verbal, (b) tabular, (c) pictórica, (d) simbólica y (e) múltiple.

- Verbal. Es aquella que hace uso del lenguaje natural para expresar la información de forma cohesionada. 
- Tabular. Es aquella en la que los estudiantes hacen uso de una tabla de datos para la organización y representación de cantidades numéricas, expresiones verbales o relaciones entre los elementos de la tarea.

- Pictórica. Es aquella que hace uso de un sistema de representación visual (generalmente un dibujo) a fin de expresar las relaciones entre datos e incógnitas en la tarea.

- Simbólica. Es aquella de carácter alfanumérico y cuya sintaxis viene descrita mediante una serie de reglas de procedimiento (Rico, 2009, p. 8).

- Múltiple. Es aquella que resulta de la combinación de dos o más sistemas de representación de los definidos anteriormente (Van Somersen, 1998).

Son múltiples las investigaciones desarrolladas sobre el pensamiento funcional. A continuación destacamos algunas de ellas. Cañadas y Fuentes (2015) realizan una investigación con 32 estudiantes de $1^{\circ}$ de educación primaria (6-7 años) en base a un problema contextualizado que involucra la función lineal $(f(x)=5 x$, teniendo en cuenta la operación de multiplicación). Uno de los principales focos de la investigación es el relativo a los sistemas de representación, obteniendo los autores como conclusiones que la representación más usado por los alumnos es el pictórico, salvo en una tarea que implica la generalización; en este caso, destaca el sistema de representación verbal. Los alumnos hacen uso también de los sistemas de representación numéricos y verbal, así como de representaciones múltiples (combinaciones de sistemas pictóricos y numéricosimbólico). En relación a las representaciones, Torres et al. (2019) destacan la verbal y la simbólica.

Ureña et al. (2019) llevan a cabo un estudio con 8 estudiantes de $4^{\circ}$ de educación primaria (8-9 años) que habían participado el año anterior en una investigación diseñada para explorar el pensamiento funcional. Por tanto, tenían experiencia previa con funciones y representaciones simbólicas y tabulares de las mismas. Los autores implementan un experimento de enseñanza en el que usan una tarea que involucra la función lineal $f(x)=$ $x+2$ (teniendo en cuenta la operación adición). Los autores se centran en la generalización y en las representaciones usadas por los estudiantes, teniendo en cuenta también la influencia de las moderaciones del investigador en las respuestas de los alumnos. 
Merino et al. (2013) estudian la habilidad de los estudiantes de $5^{\circ}$ de educación primaria (10-11 años) para generalizar en una tarea que implica la función $f(x)=2 x+2$ (teniendo en cuenta las operaciones de adición y multiplicación), considerando para ello tres aspectos: la relación funcional, la representación usada y el tipo de pregunta. Concluyen que la principal representación usada es la verbal, aunque se encuentra también evidencia de forma frecuente de la representación pictórica, seguida de la numérica. Algunos estudiantes usan la representación verbal con mayor frecuencia y los autores justifican este hecho por la comodidad para los alumnos de explicar lo que están haciendo, más aún cuando se les pidió que justificasen y explicasen sus acciones.

Merino et al. (2013) estudian la habilidad de los estudiantes de $5^{\circ}$ de educación primaria (10-11 años) para generalizar en una tarea que implica la función $f(x)=2 x+2$ (teniendo en cuenta las operaciones de adición y multiplicación), considerando para ello tres aspectos: la relación funcional, la representación usada y el tipo de pregunta. Concluyen que la principal representación usada es la verbal, aunque se encuentra también evidencia de forma frecuente de la representación pictórica, seguida de la numérica. Algunos estudiantes usan la representación verbal con mayor frecuencia y los autores justifican este hecho por la comodidad para los alumnos de explicar lo que están haciendo, más aún cuando se les pidió que justificasen y explicasen sus acciones. Pinto y Cañadas (2018) concluyen que los alumnos usan para la generalización notación algebraica y representación verbal.

Wilkie (2016) realiza una investigación con estudiantes de $1^{\circ}$ de educación secundaria (12-13 años) sin previo conocimiento de las funciones. Este estudio sugiere que las opiniones actuales sobre cuándo y cómo los niños pueden o deben aprender sobre variables y representaciones múltiples precisan de ajustes en los planes de estudio de educación matemática. Los alumnos que participaron en este estudio mostraron en una variedad de contextos capacidades para explorar aspectos más avanzados del álgebra, que tradicionalmente se han retrasado hasta que los estudiantes lleguen a la escuela secundaria. Para la autora, poco es lo que se conoce en relación del conocimiento algebraico de los estudiantes a través de sus representaciones.

\subsection{Objetivos de investigación}

El objetivo general de investigación de este trabajo es el siguiente. 
OG1. Analizar el pensamiento funcional manifestado por un grupo de estudiantes de un programa de enriquecimiento curricular de $6^{\circ}$ de educación primaria.

Abordamos este objetivo general a partir de los siguientes objetivos específicos.

OE1. Describir los valores atribuidos por los estudiantes a las variables implicadas en el problema.

OE2. Describir las estructuras establecidas por los estudiantes.

OE3. Identificar y describir representaciones usados por los estudiantes para expresar la estructura. 


\section{Capítulo 3. Metodología}

En el presente capítulo describimos los principales elementos del marco metodológico.

\subsection{Tipo de investigación}

La investigación realizada sigue un enfoque cualitativo, el cual "no pretende generalizar de manera intrínseca los resultados a poblaciones más amplias, ni necesariamente obtener muestras representativas, sino que se fundamenta más en un proceso inductivo" (p. 16) (Hernández, Fernández y Baptista, 2010). Buscamos descubrir y describir los fenómenos, buscando información en los datos. Por lo tanto, la naturaleza del presente trabajo es descriptiva. Adicionalmente, la literatura de investigación muestra que los estudios sobre aspectos como una estructura cuadrática o el uso de variable continua en dicha estructura son escasas, por lo que este trabajo es exploratorio.

\subsection{Sujetos participantes en la investigación}

La muestra es intencional, pues acudimos a un centro con el que tenemos relación y cuenta con un programa de enriquecimiento curricular. Los estudiantes que participaron en la investigación fueron los seis alumnos de $6^{\circ}$ de educación primaria (11-12 años) durante el curso 2019-2020 que pertenecían a un programa de enriquecimiento curricular en el área de matemáticas de un centro concertado de Granada.

El enriquecimiento curricular es una de las medidas para atender a la diversidad dentro del aula. De acuerdo con Benavides et al. (2004), el enriquecimiento curricular

consiste en añadir nuevos contenidos o temas que no están cubiertos por el currículo oficial o trabajar en un nivel de mayor profundidad determinados contenidos de éste. El enriquecimiento no significa avanzar en el currículo de cursos superiores, sino ampliar la estructura de los temas y contenidos abordándolos con un nivel mayor de abstracción y de complejidad. No se trata solamente de ampliar la información sobre un tema en concreto, sino de promover el uso de la investigación o del pensamiento creativo en un determinado ámbito (cómo se genera el nuevo conocimiento) y de explorar la lógica interna de éste y sus relaciones con otras áreas de conocimiento (p. $54)$.

Para pertenecer a dicho programa, los estudiantes fueron nominados como talento matemático por sus tutores por su buen rendimiento en matemáticas y demandar más 
contenidos de los trabajados habitualmente en clase. Tras la posterior evaluación por parte del departamento de orientación a través de herramientas psicométricas, fueron diagnosticados por las puntuaciones obtenidas en los correspondientes test de la forma que sigue: E1 presenta talento complejo académico; E2, talento artístico y verbal; E3 tiene talento matemático; E4 presenta talento académico y matemático; E5 talento verbal y E6 ha sido nominado por los profesores pero aún se encuentra pendiente de evaluación del departamento.

El departamento de orientación y el equipo educativo proponen que estos estudiantes trabajen en pequeños grupos de forma cooperativa durante una hora a la semana fuera del aula ordinaria en horario escolar en el programa de enriquecimiento curricular. Este programa se ofrece durante toda la etapa de educación primaria y se lleva a cabo durante todo el curso escolar.

Dada la nominación de los estudiantes como talento matemático, exponemos brevemente las características de dicho talento. El talento matemático se entiende como el desarrollo en mayor grado de características para afrontar con éxito la resolución de tareas matemáticas (Ramírez, 2012).

Las primeras investigaciones relevantes sobre el talento se encuentran en los estudios de Kruteskii (1976). El autor recopila las componentes de las habilidades matemáticas características del pensamiento matemático:

- Habilidad para percibir la estructura formal de un problema.

- Habilidad para utilizar en el pensamiento lógico las relaciones cuantitativas y espaciales, números y símbolos.

- Habilidad para pensar en símbolos matemáticos.

- Habilidad para generalizar rápida y ampliamente los objetos matemáticos, sus relaciones y sus operaciones.

- Habilidad para abreviar los procesos de razonamiento matemático y el sistema de las operaciones correspondientes.

- Flexibilidad de los procesos mentales que usan en la actividad matemática.

- Interés por clarificar, simplificar, economizar y racionalizar las soluciones.

- Reversibilidad de los procesos mentales de razonamiento. 
- Memoria matemática (para retener las relaciones matemáticas, las características, las estrategias de los argumentos y las demostraciones, los métodos de resolución de problemas y los principios de planteamientos).

- Mentalidad matemática.

Del listado de características expuestas por diferentes autores (Greenes, 1981; Kruteskii, 1976; Freiman, 2006), vemos que muchas están estrechamente relacionadas con el pensamiento funcional. Destacamos así la habilidad para utilizar en el pensamiento lógico las relaciones cuantitativas, la habilidad para pensar en símbolos matemáticos en relación con la representación, la habilidad para generalizar rápida y ampliamente los objetos matemáticos (generalización en el pensamiento funcional), sus relaciones y sus operaciones y la habilidad para abreviar los procesos de razonamiento matemático y el sistema de las operaciones correspondientes, relacionada con el establecimiento de la estructura. También mencionar la memoria para símbolos, números y fórmulas, dado que puede ponerse en juego en los aspectos relativos a la representación de la relación funcional. Destacamos también la habilidad para generalizar y el cambio fácil de una estrategia a otra, de una estructura a otra, en alusión las diferentes representaciones usadas, y la búsqueda de patrones y relaciones, la construcción nexos, lazos y estructuras matemáticas.

En las sesiones previas a las realizadas para el presente estudio, los estudiantes se encontraban completando un proyecto final del curso pasado sobre maquetas, donde el enriquecimiento era en elementos de visualización (perspectivas y orientación) y en la utilización de escalas.

La estructura del programa de enriquecimiento curricular del presente curso académico contempla en el primer trimestre actividades de visualización y análisis y diseño de juegos matemáticos; en el segundo trimestre, de resolución de problemas en equipo y en el tercer trimestre, un proyecto de investigación matemático.

Previamente al presente estudio, los estudiantes no habían trabajado las relaciones funcionales. Sí que habían tratado contenidos de enriquecimiento tales como el sentido espacial, el sentido de la medida o la resolución de problemas de forma cooperativa en dichas sesiones. La selección del centro y de los estudiantes fue intencional porque uno de los investigadores participa en la coordinación del proyecto. 


\subsection{Instrumento de recogida de información}

El instrumento de recogida de información es una tarea en forma de cuestionario que trabajamos con los estudiantes durante el desarrollo de las dos sesiones que llevamos a cabo con ellos.

Tomando como referencia la literatura expuesta y el tipo de funciones utilizadas en estudios previos, planteamos una tarea que involucra una función no lineal. En este caso, teniendo en cuenta las características de los estudiantes, proponemos la función cuadrática $A(L)=36-L^{2}$, habiéndose seleccionado segundo grado por motivos ya expuestos.

La tarea que presentamos es contextualizada y permite el uso de representación pictórica para su resolución. Dicha contextualización es la que permite trabajar con la continuidad de la variable, dado que el lado de un cuadrado puede tomar valores continuos, así como con los valores extremos de la misma. Se escoge el valor del lado como 6 por ser un número fácil de usar y la figura geométrica del cuadrado para no dificultar la realización de la tarea (los estudiantes han trabajado con anterioridad el área del cuadrado).

Describimos la tarea a continuación, diferenciada en las dos sesiones en que se realizó la recogida de datos. Dentro de cada sesión, distinguimos los enunciados, tal y como se planteó la tarea a los estudiantes; y las explicaciones adicionales que tuvimos en cuenta en el diseño de esos enunciados u otras aclaraciones desde la perspectiva metodológica.

\section{Sesión 1}

En esta primera sesión se trabaja con un cuadrado de lado 6 al que se le quita la esquina superior derecha. Preguntamos a los estudiantes por la relación existente entre el área de la figura resultante y el lado de la esquina que se elimina, siendo la misma $A(L)=36-$ $L^{2}$, donde $A(L)$ es el área de la figura resultante y $L$ es el lado de la esquina suprimida.

La tarea se compone de tres partes. Exponemos a continuación los enunciados que facilitamos a los estudiantes durante el desarrollo de la sesión, así como una breve explicación de lo que se trabaja en cada una de las partes. 


\section{Enunciados}

PARTE 1. (10 minutos)

1. Imagina un cuadrado de lado 6. Dibújalo.

2. Calcula el área del cuadrado.

PARTE 2. (15 minutos)

3. ¿Cuál es el área de un cuadradito de lado 2?

4. Dibuja en la esquina superior derecha del cuadrado de más abajo un cuadradito de lado 2. Supón que le quitamos al cuadrado de lado 6 el cuadradito de lado 2. ¿Cuál sería el área de la figura resultante?

5. ¿Cuál es el área de un cuadradito de lado 4?

6. Dibuja en la esquina superior derecha del cuadrado de más abajo un cuadradito de lado 4. Supón que le quitamos al cuadrado de lado 6 el cuadradito de lado 4. ¿Cuál sería el área de la figura resultante?

\section{PARTE 3. (15 minutos)}

7. Dibuja en la esquina superior derecha un cuadradito. ¿Cómo explicarías a un amigo tuyo cómo calcular el área de un cuadrado si no podemos saber cuánto mide su lado?

8. Imagina ahora que el lado del cuadradito valiera L. ¿Podríamos ahora calcular su área?

9. Supón ahora que le quitamos al cuadrado de lado 6 el cuadradito de lado L. ¿Cuál sería el área de la figura resultante?

10. ¿Crees que puede tomar valores decimales? ¿Y negativos?

11. ¿Cuál sería el valor más pequeño para el lado del cuadradito? ¿Cuál sería el valor más grande para el lado del cuadradito?

12. ¿Cuál será el valor más pequeño de la figura resultante? ¿Cuál será el valor más grande la de la figura resultante?

13. ¿Qué valores crees que podría tomar L? 


\section{Explicación}

PARTE 1. (10 minutos)

Se trabaja con un cuadrado de lado 6 y se le pide al estudiante que calcule su área. En esta parte, se trabaja el concepto de área y su cálculo y se busca introducir al estudiante el contexto de la tarea (figura 1).

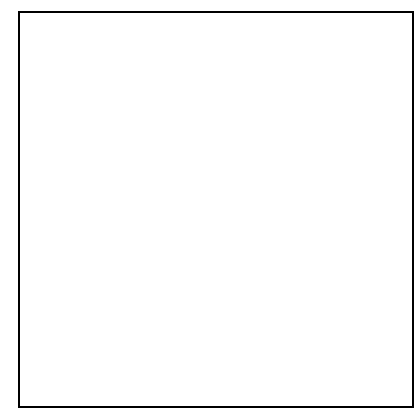

Figura 1. Esquema de la parte 1

\section{PARTE 2. (15 minutos)}

Se trabaja con un cuadrado de lado 6 del que se eliminan dos cuadraditos en la esquina superior derecha de lados 2 y 4 , respectivamente. Se le pide al estudiante que dibuje la situación y que determine el área de la figura resultante de la sustracción de las esquinas mencionadas (figura 2).
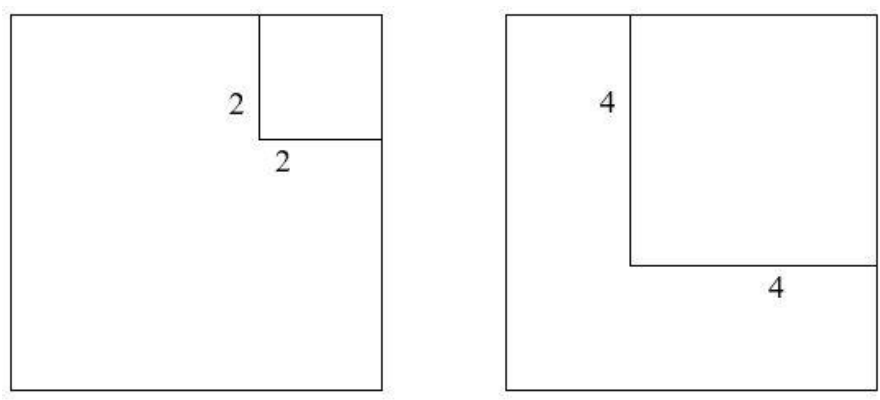

Figura 2. Esquema de la parte 2

PARTE 3. (15 minutos)

Se trabaja con un cuadrado de lado 6 del que se elimina un cuadradito en la esquina superior derecha de lado desconocido. Se pide al estudiante que dibuje la situación y determine el área de la figura resultante de la sustracción de la esquina mencionada (figura $3)$. 


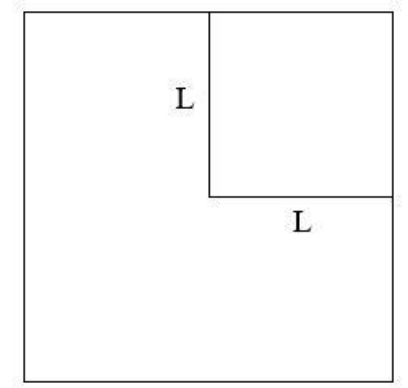

Figura 3. Esquema de la parte 3

En esta parte se busca generalizar las dos anteriores. Ya conocido el procedimiento para calcular el área, los estudiantes deben establecer la estructura entre el lado de la esquina y el área de la figura resultante. Analizamos también la representación usada, si es única o no y si los estudiantes distinguen el hecho de que a más longitud del lado, menor área resultante (variables inversas).

Les preguntamos a los estudiantes si L (lado) puede tomar valores decimales o negativos en esta parte, trabajando así la variable continua. También se trabajan los valores extremos de la variable $\left(L_{\min }=0, L_{\max }=6\right)$, los valores extremos del área resultante $\left(A_{\min }=0, A_{\max }=36\right) \mathrm{y}$ si los estudiantes establecen o no un intervalo de valores para ambos o tan sólo dan valores concretos.

\section{Sesión 2}

La segunda sesión fue una entrevista individual con cada uno de los estudiantes. La llevamos a cabo dos investigadores del equipo, siendo uno de ellos el entrevistador y el otro, el encargado de grabaciones y observación. Les planteamos dos tareas similares a la de la primera sesión: un cuadrado al que se le sustraen cuatro esquinas iguales y un cuadrado al que se le sustraen dos esquinas opuestas, no necesariamente iguales. En la primera tarea, la relación es $A(L)=36-4 L^{2}$, donde $A(L)$ es el área de la figura resultante y $L$ es el lado de la esquina suprimida; en la segunda, $A(L, J)=36-L^{2}-J^{2}$, donde $A(L, J)$ es el área de la figura resultante, $L$ es el lado de una de las esquinas suprimidas y $J$ es el lado de la otra esquina. La entrevista individual tuvo una duración máxima de media hora, aunque hubo estudiantes que la realizaron en menor tiempo.

La tarea se compone de dos partes. Exponemos a continuación los enunciados que facilitamos a los estudiantes durante el desarrollo de la sesión, así como una breve explicación de lo que se trabaja en cada una de las partes. 


\section{Enunciados}

PARTE 4. (10 minutos)

14. Imagina ahora que dibujamos un cuadradito de lado $L$ en cada esquina del cuadrado de lado 6. Dibuja la figura.

15. ¿Cuál sería el área de la figura resultante al quitar las cuatro esquinas?

16. ¿Crees que puede tomar valores decimales? ¿Y negativos?

17. ¿Cuál sería el valor más pequeño para el lado del cuadradito? ¿Cuál sería el valor más grande para el lado del cuadradito?

18. ¿Qué valores podría tomar L en esta ocasión?

\section{PARTE 5. (10 minutos)}

19. Imagina ahora que dibujamos dos cuadraditos en esquinas opuestas del cuadrado de lado 6. Dibuja la figura.

20. Si sabemos que los lados de los dos cuadraditos no son iguales, ¿cómo podemos simbolizar el valor de los lados?

21. ¿Cuál sería el área de la figura resultante de quitar los dos cuadraditos?

22. ¿Crees que puede tomar valores decimales? ¿Y negativos?

23. ¿Cuál sería el valor más pequeño para el lado de un cuadradito? ¿Y del otro?

24. ¿Cuál sería el valor más grande para el lado de un cuadradito? ¿Y del otro?

25. ¿Qué valores podría tomar uno de los cuadraditos? ¿Y el otro?

\section{Explicación}

\section{PARTE 4. (10 minutos)}

Se trabaja con un cuadrado de lado 6 del que se elimina un cuadradito por esquina de lado desconocido. Se pide al estudiante que dibuje la situación y determine el área de la figura resultante de la sustracción de las esquinas mencionadas (figura 4). A esta tarea será a la que nos referiremos como tarea de las 4 esquinas. 


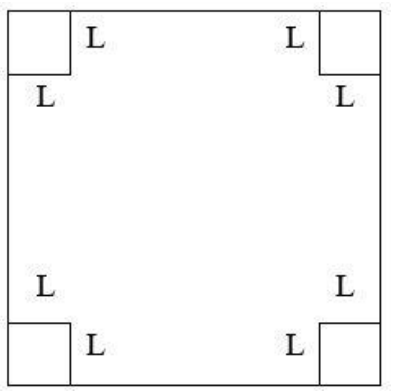

Figura 4. Esquema de la parte 4

Los estudiantes deben establecer la relación entre el lado de la esquina y el área de la figura resultante de eliminar cuatro esquinas. Analizamos también la representación usada, si es única o no y si los estudiantes distinguen el hecho de que a más lado, menor área resultante (variables inversas). Se tiene también en cuenta si se hace uso de la misma variable para todas las esquinas.

Les preguntamos a los estudiantes si L (lado) puede tomar valores decimales o negativos en esta parte, trabajando así la variable continua. También se trabajan los valores extremos de la variable $\left(L_{\min }=0, L_{\max }=3\right)$, los valores extremos del área resultante $\left(A_{\min }=0, A_{\max }=36\right)$ y si los estudiantes establecen o no un intervalo de valores para ambos o tan sólo dan valores concretos.

\section{PARTE 5. (10 minutos)}

Se trabaja con un cuadrado de lado 6 del que se eliminan dos cuadraditos en esquina opuestas de lados desconocidos, sabiendo que los cuadraditos no tienen por qué tener lados de igual medida. Se pide al estudiante que dibuje la situación y determine el área de la figura resultante de la sustracción de las esquinas mencionadas (figura 5). A esta tarea será a la que nos referiremos como tarea de las 2 esquinas.

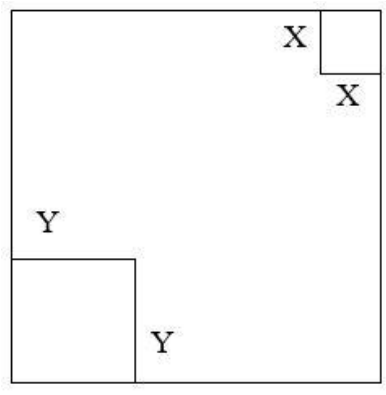

Figura 5. Esquema de la parte 5

Los estudiantes deben establecer la estructura entre el lado de la esquina y el área de la figura resultante de eliminar dos esquinas opuestas. Analizamos también la forma de 
representación usada, si es única o no y si los estudiantes distinguen el hecho de que a mayor longitud del lado, menor área resultante (variables inversas). Se tiene también en cuenta si se hace uso de diferentes variables para designar cada esquina: si se usan letras diferentes, si se dan valores diferentes, si las relacionan... Les preguntamos a los estudiantes si L (lado) puede tomar valores decimales o negativos en esta parte, trabajando así la variable continua. También se trabajan los valores extremos de la variable, la relación entre los lados de ambas esquinas (la suma de ambos lados debe ser igual a 6 en caso de rellenarse todo el lado), los valores extremos del área resultante $\left(A_{\min }=0, A_{\max }=36\right)$ y si los estudiantes establecen o no un intervalo de valores para ambos o tan sólo dan valores concretos.

En las sesiones de enriquecimiento posteriores a esta investigación, se trabajó con los estudiantes el volumen de las cajas generadas a partir del siguiente desarrollo plano (figura 6):



Figura 6. Desarrollo plano usado para generar cajas

Dicho desarrollo se corresponde con la figura 4, con la que los estudiantes ya habían trabajado previamente. Se busca conocer el volumen de dicha caja y optimizarlo en función de los valores que pueda tomar la variable lado. Para ello, en las sesiones se trabajó con los estudiantes con una tabla de valores (representación tabular de la relación que existe entre el volumen de la caja y el valor del lado suprimido en la base). Dicho volumen se calculó haciendo uso de medidas directas (usando material multibase para el relleno) e indirectas (fórmula, representación simbólica de la estructura). Se buscaba así enriquecer el concepto de volumen. 


\subsection{Recogida de la información}

Llevamos a cabo la recogida de datos en dos sesiones, ambas llevadas a cabo por dos investigadores, la autora de este trabajo y el tutor. La tutora de los estudiantes también estuvo presente pero no intervino. La autora de este trabajo y el tutor guiaron la puesta en común ayudaron a los estudiantes, prestando su ayuda si no sabían cómo responder a alguna de las preguntas planteadas en la tarea en caso de no comprender el estudiante lo que se pide, por ejemplo.

Ambas sesiones fueron llevadas a cabo en horario de clases de enriquecimiento, en un espacio habilitado por el centro escolar y grabadas en vídeo. Por tanto, contamos con grabaciones de las dos sesiones, así como con el material escrito de los estudiantes.

Durante la primera sesión los estudiantes realizaron la tarea individualmente para luego ser puesta en común en gran grupo y se trabajaron las partes 1, 2 y 3 de la tarea. Se trabajó parte por parte, de forma que previamente a desarrollar una de ellas, ya se había comentado en gran grupo la anterior y las conclusiones sacadas por los estudiantes habían quedado claras a los mismos y a los investigadores.

Durante la segunda sesión se realizó una entrevista individual a cada uno de los estudiantes, de una duración aproximada de media hora cada una. En dicha entrevista se les preguntaba a los estudiantes por un breve recordatorio de la sesión anterior, para recordar las ideas, y se les proponían las partes 4 y 5 de la tarea.

En cada una de las preguntas se pide al estudiante que explique o justifique la respuesta que da. Hay ocasiones en las que los investigadores aclaran o preguntan al estudiante ante un error cometido o ante una situación en la que el estudiante no sabe cómo continuar.

\subsection{Categorías de análisis}

La unidad de análisis del presente trabajo son las respuestas dadas por los estudiantes. Tenemos en cuenta en cuenta tres momentos diferenciados en el desarrollo de la investigación: (a) el trabajo individual realizado por cada estudiante en la primera sesión, (b) la puesta en común de dicha sesión y (c) la entrevista de la segunda sesión.

Establecemos cuatro categorías de análisis, tomando como referencia para el establecimiento de las mismas los objetivos de investigación ya planteados: 
- Valores extremos de la variable.

- Valores de la variable independiente.

- Estructuras.

- Representación.

\subsection{Organización del análisis de datos}

El primer paso realizado en el análisis de datos fue la transcripción de la sesión grupal y de las entrevistas individuales llevadas a cabo. Dichas transcripciones, junto con las respuestas escritas de los estudiantes, constituyen los datos con los que contamos para la investigación. A continuación, organizamos las respuestas de los estudiantes con base en las categorías de análisis descritas y realizamos una descripción de los resultados. Para ello, presentamos resúmenes de resultados a través de tablas que atienden a los elementos de los objetivos de investigación, las describimos en general y detallamos algunos casos representativos por presentarse en varios estudiantes o significativos en algunos de los aspectos abordados en las preguntas de investigación. 


\section{Capítulo 4. Análisis de datos y resultados}

Llevamos a cabo un análisis de datos cualitativo. Recordamos que las categorías son las siguientes, que ya hemos descritos en el capítulo de metodología:

- Valores extremos de la variable.

- Valores de la variable independiente.

- Estructura.

- Representación.

Cada una de estas categorías se observa en tres momentos de la recogida de datos, para cada estudiante: (a) en el trabajo individual de la sesión grupal, (b) en la puesta en común de dicha sesión y (c) en la entrevista.

Se detallan a continuación los resultados obtenidos, especificándose para cada momento citado.

\subsection{Trabajo en el cuestionario}

Los resultados que se muestran a continuación se han extraído de las respuestas escritas en el cuestionario por parte de los estudiantes.

\section{Valores extremos de las variables}

En la tabla 1 presentamos el resumen de valores extremos que los estudiantes exponen en sus trabajos individuales. Para ello, mostramos los valores máximos y mínimos dados por los estudiantes.

Tabla 1.

Valores extremos en el trabajo en el cuestionario

\begin{tabular}{|c|c|c|c|c|}
\hline \multirow[b]{2}{*}{ Estudiante } & \multicolumn{2}{|c|}{ Valor mínimo } & \multicolumn{2}{|c|}{ Valor máximo } \\
\hline & Lado & Área & Lado & Área \\
\hline E1 & 1 & - & 5 & - \\
\hline E2 & 0.1 & 0 & Infinito & Infinito \\
\hline E3 & 2 & 4 & 6 & 36 \\
\hline E4 & 1 & 11 & 5 & 35 \\
\hline E5 & $1 \times 1$ & 1 & $2.5 \times 2.5$ & 4 \\
\hline E6 & $\begin{array}{l}\text { No se sabe por } \\
\text { ser infinito }\end{array}$ & No se sabe & $\begin{array}{l}\text { No se sabe por } \\
\text { ser infinito }\end{array}$ & No se sabe \\
\hline
\end{tabular}


Tabla 1.

Valores extremos en el trabajo en el cuestionario

\begin{tabular}{|c|c|c|}
\hline & Valor mínimo & Valor máximo \\
\hline Estudiante & Lado & Lado \\
\hline
\end{tabular}

De los seis estudiantes, ninguno de ellos estableció el valor mínimo de la variable y solamente E3 estableció de forma correcta el valor máximo. En cuanto a los valores extremos del área, E2 establece el mínimo y E3 el máximo, pero los restantes estudiantes no determinaron dichos valores de forma correcta. Llama la atención que un estudiante (E5), expresó los valores extremos de la misma variable en forma de producto, probablemente indicando de esta forma el área como producto del valor del lado, y que otro (E6), mencionase no saberse los valores máximos y mínimos de las variables, en el caso del lado por "ser infinito".

Podemos ver que hay dos estudiantes (E1 y E4) que dieron los mismos valores extremos para la variable lado: 1 y 5 . Este hecho era esperable: denota claramente que hicieron un uso totalmente discreto de la variable, no considerando además los valores extremos del intervalo. En primera instancia, hicieron uso de valores discretos y no consideraron los extremos del intervalo por pensar que no tenía sentido que $L=0$ (no se recorta nada del cuadrado) y que $L=6$ (se toma todo el cuadrado), por lo que tomaron los dos valores siguientes, 1 (el menor) y 5 (el mayor), asignándole así valores discretos a la variable $L$.

\section{Valores de la variable independiente}

En la tabla 2 presentamos el resumen del trabajo individual de los estudiantes en relación al tipo de valores que toma la variable. Marcamos con "sí" cuando un estudiante considera que la variable puede tomar valores decimales o negativos y como "no" cuando establece lo contrario.

Tabla 2.

Valores en el trabajo en el cuestionario

\begin{tabular}{lll}
\hline Estudiante & Carácter decimal & Carácter negativo \\
\hline E1 & - & - \\
E2 & Sí & No \\
E3 & No & No
\end{tabular}


Tabla 2.

Valores en el trabajo en el cuestionario

\begin{tabular}{lll}
\hline Estudiante & Carácter decimal & Carácter negativo \\
\hline E4 & No & No \\
E5 & Sí & No \\
E6 & Sí & Sí \\
\hline
\end{tabular}

Nota. - = no hay información disponible.

E1 no ofreció información. De los cinco restantes, tres (E2, E5 y E6) reconocieron que puede tomar valores decimales y cuatro (E2, E3, E4 y E5), que no puede tomar valores negativos. De los cinco estudiantes que responden, cuatro justificaron sus respuestas.

\section{Estructuras y representación}

En la tabla 3 presentamos el resumen del trabajo individual de los estudiantes en relación a la estructura y la representación de la misma. Marcamos con "sí" cuando un estudiante considera que la variable puede tomar valores decimales o negativos y como "no" cuando establece lo contrario.

Tabla 3.

Estructuras y representación en el trabajo en el cuestionario

Estructura

\begin{tabular}{llll}
\cline { 2 - 3 } Estudiante & \multicolumn{2}{c}{ Casos particulares } & \multicolumn{2}{c}{ Caso general } & Representaciones \\
\hline E1 & Sí & No & - \\
E2 & No & $36-L^{*}$ & Verbal y pictórica \\
E3 & Sí & No & - \\
E4 & Sí & No & - \\
E5 & No & $36-L^{*}$ & Verbal \\
E6 & No & $36-L^{*}$ & Verbal \\
\hline
\end{tabular}

Nota. $*$ = Respuestas incorrectas de los estudiantes; - = no hay información disponible.

De los seis estudiantes, tres de ellos (E1, E3 e E4) no establecieron la estructura, sino que dieron valores concretos a la variable. De los otros tres, dos (E5 y E6) establecieron la estructura de forma verbal, pero sin mencionar la variable. Es decir, establecieron una estructura verbal no equivaliendo ésta a la lectura de la función simbólica. E2 estableció la estructura de forma verbal y pictórica, sin ser tampoco correcta por establecer los tres la estructura $36-L$. 


\subsection{Puesta en común}

Los resultados que se muestran a continuación se han extraído de las transcripciones realizadas de las grabaciones de las sesiones. No disponemos de información de todos los estudiantes dado que no todos ellos respondieron todas las preguntas durante la puesta en común.

\section{Valores extremos de las variables}

En la tabla 4 presentamos el resumen de valores extremos que los estudiantes exponen en la puesta en común. Para ello, se muestran los valores máximos y mínimos dados por los estudiantes.

\begin{tabular}{|c|c|c|c|c|}
\hline \multirow[b]{2}{*}{ Estudiante } & \multicolumn{2}{|c|}{ Valor mínimo } & \multicolumn{2}{|c|}{ Valor máximo } \\
\hline & Lado & Área & Lado & Área \\
\hline E1 & - & - & - & - \\
\hline E2 & 0 & - & 6 & 36 \\
\hline E3 & - & - & - & 36 \\
\hline E4 & - & - & - & 36 \\
\hline E5 & - & - & - & - \\
\hline E6 & 0 & 0 & 6 & 36 \\
\hline
\end{tabular}

Para el establecimiento de los valores expuestos en la tabla 4, los estudiantes precisaron de estímulos por parte de los investigadores. Un ejemplo lo observamos en el siguiente fragmento, dicho por uno de los investigadores: “fijaos: la L no puede ser más grande que el cuadrado de lado 6", "podría ser 6 y que no hubiera nada, igual que la L más pequeña podría ser 0 , que no le estamos quitando nada”, “el área no sería más grande que 36”.

Inicialmente, el investigador preguntó por los extremos de la variable. En este caso, todos los estudiantes respondieron que si $L=0, A=36$ y que si $L=6, A=0$, estableciendo uno de los estudiantes (E2) de forma natural el intervalo de valores: "todos no pasando del 0 y $6 "$. 
Dos de los estudiantes no respondieron durante la puesta en común. De los cuatro restantes, E2 estableció un intervalo, [0,6] para los valores del lado y E6 reconoció los valores máximo y mínimo pero no estableció un intervalo. También justificó que para el valor máximo del lado, no hay cuadrado grande.

En cuanto a los valores del área, E2 e E4 reconocieron el área máxima y E6 reconoció los valores máximo y mínimo con los valores mínimo y máximo respectivamente que anteriormente había establecido para el lado.

Es interesante notar que E2 reconoció que, en caso de ser el área mínima, el cuadradito que se ha eliminado tiene área máxima cuando dijo "tendría que ser 6 , porque 6 por 6,36 , y sería el área completa". El último de ellos, E3, confundió el área con el lado al decir que el valor más grande del área es 36.

\section{Valores de la variable independiente}

En la tabla 5 presentamos el resumen de la puesta en común de los estudiantes en relación al tipo de valores que toma la variable. Marcamos con "sí" cuando un estudiante considera que la variable puede tomar valores decimales o negativos y como "no" cuando establece lo contrario.

Tabla 5.

\begin{tabular}{lll}
\multicolumn{3}{l}{ Valores en la puesta en común } \\
\hline Estudiante & Carácter decimal & Carácter negativo \\
\hline E1 & - & - \\
E2 & Sí & No \\
E3 & - & - \\
E4 & No & No \\
E5 & - & No \\
E6 & - & - \\
\hline Nota. - = no hay información disponible.
\end{tabular}

En esta ocasión, solamente hay información de tres de los 6 estudiantes. Uno de ellos (E2) respondió correcta y justificadamente (en el trabajo individual no había incluido justificación): "puedes poner la L un número, 3.5”, “es una medida negativa, que no positiva". De los otros dos que respondieron, ambos lo hicieron correctamente con respecto al carácter negativo de la variable pero no con respecto al carácter decimal. En este caso, uno de ellos ni siquiera comentó esa parte. 


\section{Estructuras}

En la puesta en común distinguiremos los resultados obtenidos en cada una de las preguntas. En la primera de ellas, en las que pedimos a los estudiantes que explicasen a un amigo cómo calcular el área de un cuadrado al que suprimimos una esquina si no conocemos el valor de su lado, son cinco los estudiantes que respondieron. Dos de ellos, E3 y E6, mencionaron el uso de la regla para obtener el valor concreto de la esquina y poder calcular el área. Establecieron la estructura al exponer que restarían el área del cuadrado pequeño al área del cuadrado grande, pero tuvieron la necesidad de conocer el dato concreto: "pues yo he puesto multiplicamos 6 por 6 y el resultado se lo restamos. Con la regla, calculamos el cuadrado y se lo restamos a 36" (E3), "he puesto yo lo mediría con una regla y a partir de ahí lo haría restándole el pequeño al grande” (E6). E1 y E5 expusieron que sin conocer el número del cuadrado no se puede calcular el área. Su postura es similar a la de E6 y E3, por necesitar el valor concreto del lado para calcular el área, pero ellos no mencionaron el uso de la regla para solventar esto. Por su parte, E2 expresó la estructura de forma verbal, sin tener en cuenta la regla, cuando dijo "yo lo he puesto suponiendo que no tengo regla. He dicho para calcular el área hay que multiplicar la altura y la base del cuadrado pequeño, después la del cuadrado grande y los resultados hay que restarle el resultado del grande restárselo al cuadrado pequeño y eso es el resultado del área”. En general, puede decirse que los cinco establecieron una relación entre las dos variables al decir que necesitaban conocer el lado de la esquina para poder calcular el área.

En la segunda pregunta, en la que pedimos a los estudiantes que calculasen el valor del área de un cuadrado en caso de valer L el lado del mismo, es de los mismos cinco estudiantes de quien se obtuvo información. Es un primer momento, E1 sostuvo que deben tomarse valores concretos para calcular el área. Ante la mención de la letra L, E3 dijo que calcular el área sí es posible "porque L es un número, pero en letra”. E2, E5 y E6 fueron más allá y establecieron que el área pequeña es $\mathrm{L} \times \mathrm{L}$ y que, restándosela al grande, obtendrían el área de la figura resultante. Es interesante notar que E5, si bien en la pregunta anterior no calculaba el área, ante la introducción de la variable L sí que respondió de manera correcta. Tras la ayuda y puesta en común por parte de los investigadores, todos llegan a comprender el cálculo del área, expresado de forma verbal. 
En la última pregunta, en la que pedimos a los estudiantes que calculasen el valor del área de la figura resultante en caso de valer L el lado de la esquina, fueron de nuevo cinco los estudiantes de los que se dispone información. En esta parte de la puesta en común, uno de los investigadores guía la misma mediante el uso de preguntas relativas a los comentarios y respuestas que van ofreciendo los estudiantes: "vamos a discutirlo un momentito. ¿Qué opináis de la respuesta que ha dado vuestro compañero? ¿Estáis de acuerdo?”, “si queremos calcular el área del cuadrado, ¿cómo lo hacemos?”. E6, E2 y E5 establecieron $36-L$ como estructura y E3 persistió en el uso de valores particulares. Los investigadores ayudaron a los estudiantes a establecer la estructura de forma simbólica mediante pasos, comprobando al final que todos ellos la entendieron y eran capaces de establecerla. Para ello, hicieron uso de representaciones pictóricas y simbólicas en la pizarra del aula (figura 7), mientras que comentaban con los estudiantes las respuestas y guiaban la puesta en común. Aunque los seis llegaron a esto, solamente tres de ellos (E2, E3 y E6) establecieron por sí solos que el área pequeña es L×L (aunque en la estructura establecieron $36-L)$.



Figura 7. Representaciones pictórica y simbólica usadas por los investigadores

Destaca también el hecho de que uno de los estudiantes usara la expresión "doble L" para representar el área del cuadrado pequeño. E2 realizó bien el cálculo, pero no fue capaz de simbolizarlo algebraicamente: "yo lo que he hecho, en vez de representarlo así, ha sido poner L por L igual, he hecho un círculo y lo he mandado a la resta”, por lo que usó una representación que podríamos denominar como pictórica.

\section{Representación}

En la tabla 6 presentamos el resumen de la puesta en común de los estudiantes en relación a la representación. 
Tabla 6.

Representación en la puesta en común

\begin{tabular}{ll}
\hline Estudiante & \multicolumn{1}{c}{ Representación } \\
\hline E1 & - \\
E2 & Verbal, simbólica y pictórica \\
E3 & Verbal \\
E4 & Verbal \\
E5 & Verbal y simbólica \\
E6 & Verbal y simbólica \\
\hline Nota. - = no hay información disponible.
\end{tabular}

En esta ocasión, cinco de los seis estudiantes contestaron a la pregunta. Todos ellos establecieron la estructura haciendo uso de una representación verbal, utilizando E5, E6 y E2 también la representación simbólica. Este último hizo también uso de la representación pictórica.

\subsection{Entrevista}

Los resultados que se muestran a continuación se han extraído de las respuestas escritas en el cuestionario por parte de los estudiantes y de las transcripciones realizadas de las grabaciones de la entrevista llevada a cabo en la segunda sesión.

\section{Valores extremos de las variables}

Para establecer los valores extremos de la variable y del área, en la entrevista se establecen dos situaciones diferentes: se eliminan 4 esquinas iguales (parte 4) y se eliminan 2 esquinas opuestas diferentes (parte 5). Con base en esto, se presentan los resultados obtenidos.

En la tabla 7 presentamos el resumen de valores extremos que los estudiantes exponen en sus entrevistas en relación con la tarea de las 4 esquinas (parte 4). Para ello, se muestran los valores máximos y mínimos dados por los estudiantes y el establecimiento de intervalos por parte de los estudiantes, pues en esta tarea llegan a establecer intervalos. 
Tabla 7.

Valores extremos en la tarea de las 4 esquinas en la entrevista

\begin{tabular}{|c|c|c|c|c|c|}
\hline \multirow[b]{2}{*}{ Estudiante } & \multicolumn{2}{|c|}{ Valor mínimo } & \multicolumn{2}{|c|}{ Valor máximo } & \multirow[b]{2}{*}{ Intervalo } \\
\hline & Lado & Área & Lado & Área & \\
\hline E1 & $0 *$ & - & $6 *$ & 36 & {$[0,3]^{*}$} \\
\hline E2 & 0 & 0 & $3 *$ & 36 & $0<\mathrm{L}<6$ \\
\hline E3 & $1 *$ & 20 & $2 *$ & 36 & - \\
\hline E4 & Cercano a 0 & $0 *$ & $3 *$ & 5.9 & $\begin{array}{l}1,2,3,4,5 \\
(0.1,5.9)\end{array}$ \\
\hline E5 & Más de cero, 1 & 12 & $3 *$ & $36^{*}$ & $0<\mathrm{L}<3$ \\
\hline E6 & 0 & $0 *$ & $2 *$ & 36 & - \\
\hline
\end{tabular}

Nota. ${ }^{*}=$ Respuestas de los estudiantes con ayuda por parte de los investigadores; - = no hay información disponible.

Con respecto al valor mínimo de la variable, E1, E2 y E6 respondieron de forma correcta, precisando el primero de ellos de estímulos por parte de la entrevistadora (es la entrevistadora la que guía la entrevista mediante preguntas como “¿cuánto sobra aquí?”, “¿puedo poner otro número que no sea 2?”, “¿cómo poco cuánto puede valer?”) y obteniendo el resultado mediante ensayo-error. Es curioso notar que E5 estableció como valor mínimo "más de cero, 1", pero luego sí contempló que la variable pudiera tomar valores decimales. En cuanto al valor máximo, todos precisaron de ayuda y fueron E2, E4 y E5 quienes lo establecieron bien. Todos los estudiantes respondieron al inicio que el valor máximo es 6 , sin tener en cuenta la presencia en esta ocasión de dos esquinas por cada lado del cuadrado.

En relación al valor mínimo del área, E1 no respondió. De los restantes, E2, E4 y E6 respondieron correctamente. Para el valor máximo del área todos salvo E4 lo identificaron bien.

Tuvimos en cuenta si los estudiantes establecen un intervalo de valores que de forma natural (sin necesidad de ayuda o estímulos por parte de los investigadores) para los valores de la variable y del área. E1 y E5 establecieron que el valor del lado está comprendido entre cero y tres (a pesar que de E1 no establece bien el valor máximo de la variable). E2 expuso que la variable está comprendida entre cero y seis y E4 estableció un intervalo que va desde 0.1 a 5.9, ya que estableció como valor mínimo "un valor cercano a cero". 
En la tabla 8 presentamos el resumen de valores extremos que los estudiantes exponen en sus entrevistas en relación con la tarea de las 2 esquinas (parte 5). Para ello, se muestran los valores máximos y mínimos dados por los estudiantes.

\begin{tabular}{|c|c|c|c|c|}
\hline \multirow{2}{*}{$\begin{array}{c}\text { Estudiant } \\
\mathrm{e}\end{array}$} & \multicolumn{2}{|c|}{ Valor mínimo } & \multicolumn{2}{|c|}{ Valor máximo } \\
\hline & Lado & Área & Lado & Área \\
\hline E1 & - & - & - & 36 \\
\hline E2 & 0 & - & 4 & 6 \\
\hline E3 & 1 & - & 4 & - \\
\hline E4 & 0.1 & 0 & 5.9 & $36^{*}$ \\
\hline E5 & 0 & - & 5 & - \\
\hline E6 & 0 & - & 6 & - \\
\hline
\end{tabular}

Con respecto al valor mínimo de la variable, E3 e E4 respondieron de forma incorrecta. En relación al valor mínimo del área, es E4 el que respondió y lo hizo correctamente. Para el valor máximo del área, E1 e E4 lo identificaron bien, pero E2 no. El resto no ofrecieron respuesta.

E2 expuso como valor máximo 4 justificándolo mediante el uso de áreas: si vale 4, 4 . $4=16,16+16=32$, que no excede a 36 (que sería el área total del cuadrado grande); si vale $5,5 \cdot 5=25,25+25=50$, que excede a 36 .

\section{Valores de la variable independiente}

En la tabla 9 presentamos el resumen de la entrevista de los estudiantes en relación al tipo de valores que toma la variable. Marcamos con "sí" cuando un estudiante considera que la variable puede tomar valores decimales o negativos y como "no" cuando establece lo contrario. 
Tabla 9.

Valores en la entrevista

\begin{tabular}{|c|c|c|c|c|}
\hline \multirow[b]{2}{*}{ Estudiante } & \multicolumn{2}{|c|}{ Tarea de las 4 esquinas } & \multicolumn{2}{|c|}{ Tarea de las 2 esquinas } \\
\hline & $\begin{array}{l}\text { Carácter } \\
\text { decimal }\end{array}$ & $\begin{array}{l}\text { Carácter } \\
\text { negativo }\end{array}$ & $\begin{array}{l}\text { Carácter } \\
\text { decimal }\end{array}$ & $\begin{array}{l}\text { Carácter } \\
\text { negativo }\end{array}$ \\
\hline E1 & - & No & - & - \\
\hline E2 & Sí & No & Sí & - \\
\hline E3 & Sí & No & Sí & No \\
\hline $\mathrm{E} 4$ & Sí & No & Sí & No \\
\hline E5 & Sí & No & Sí & No \\
\hline E6 & Sí & No & Sí & No \\
\hline
\end{tabular}

Nota. - = no hay información disponible.

Planteamos en la entrevista dos cuestiones relacionadas con el tipo de valor de la variable.

En la primera de ellas (parte 4), los seis estudiantes respondieron, realizándolo cinco de ellos de forma correcta (uno con ayuda) y justificada. El restante (E1) dudó en cuanto al carácter decimal de la variable. En la segunda (parte 5), respondieron cinco, haciéndolo cuatro de ellos de forma correcta y justificada. El quinto de ellos no justificó y no respondió al carácter negativo de la variable (E2).

\section{Estructuras}

Para establecer la estructura, en la entrevista se plantearon dos tareas diferentes: se eliminan 4 esquinas iguales (parte 4) y se eliminan 2 esquinas opuestas diferentes (parte 5). En base a esto, se presentan los resultados obtenidos.

En la tabla 10 se indica si los estudiantes identifican o no las estructuras en sus entrevistas en relación con la tarea de las 4 esquinas. Identificamos con "sí" cuando un estudiante estableció la estructura (de forma correcta o no) y como "no" cuando no la estableció. 
Tabla 10.

Estructuras en la tarea de las 4 esquinas en la entrevista

\begin{tabular}{lll}
\hline \multirow{2}{*}{ Estudiante } & \multicolumn{2}{c}{ Estructura } \\
\cline { 2 - 3 } & Casos particulares & \multicolumn{1}{c}{ Caso general } \\
\hline E1 & $36-4,36-16$ & No \\
E2 & No & $36-4 L^{2}$ \\
E3 & No & $36-4 L^{2}$ \\
E4 & No & $36-L(*)$ \\
E5 & No & $36-4 L^{*}(*)$ \\
E6 & No & $36-4 L^{2}$ \\
\hline Nota. $*=$ Respuestas de los estudiantes con ayuda por parte de los \\
investigadores. $(*)=$ Respuestas incorrectas de los estudiantes.
\end{tabular}

En cuanto a la tarea relativa a las cuatro esquinas (parte 4), cinco de los seis estudiantes establecieron la estructura y E1, el restante, hizo uso de casos particulares, sin llegar a generalizar, para establecer una estructura particular de carácter verbal. E2, E3 e E4 establecieron la relación verbal de forma correcta, pero tuvieron problemas al expresarla en simbolismo algebraico. Establecieron $36-L$ al no ser capaces de agrupar $L \times L$ de forma correcta a pesar de exponer que el área del cuadrado pequeño se calcula de dicha forma. También omitieron el uso del 4 en el simbolismo algebraico, a pesar de mencionarlo en la estructura verbal. E5 sí que introdujo el 4 pero estableció $36-4 L$, al tener problemas con la manipulación de los términos. E6 estableció la estructura de forma correcta.

En relación al uso de la misma letra para las cuatro esquinas, todos ellos salvo E1 usaron sólo una letra. Los seis expresaron en sus diferentes relaciones que el área del cuadrado pequeño debe ir multiplicada por 4, aunque E2 requirió de ayuda para ello cuando la entrevistadora le preguntó “¿todos los resultados son los mismos o son diferentes?”, ‘por cuánto lo multiplicamos?”.

Las estructuras de E4 (al eliminar el factor 4 en la expresión) y de E5 (precisando incluso de ayuda para ello) no fueron correctas pero la de E6 sí, expresando este último la estructura de forma clara y segura. 
En la tabla 11 se indica si los estudiantes identifican o no las estructuras en sus entrevistas en relación con la tarea de las 2 esquinas. Identificamos con "sí" cuando un estudiante estableció la estructura (de forma correcta o no) y como "no" cuando no la estableció.

Tabla 11.

Estructuras en la tarea de las 2 esquinas en la entrevista

\begin{tabular}{lll}
\hline \multirow{2}{*}{ Estudiante } & \multicolumn{2}{c}{ Casos particulares } \\
\cline { 2 - 3 } & No & \multicolumn{1}{c}{ Caso general } \\
E2 & No & $36-L=32,36-J=30 *(*)$ \\
E3 & No & $36-L^{2}-J^{2}$ \\
E4 & No & $36-L^{2}-J^{2}$ \\
E5 & No & $36-L-J\left(^{*}\right)$ \\
E6 & No & $36-L^{2}-J^{2}$ \\
\hline Nota. $*=$ Respuestas de los estudiantes con ayuda por parte de los investigadores. $\left(^{*}\right)=$ \\
Respuestas incorrectas de los estudiantes.
\end{tabular}

En relación a la tarea que implican dos esquinas diferentes (parte 5), los seis estudiantes lograron establecer la estructura sin hacer uso de casos particulares ninguno de ellos. E1 precisó de ayuda del entrevistador para establecerlas. Aun así, las que estableció no son correctas, dado que estableció dos relaciones independientes. E2 estableció la siguiente estructura verbal "tendría que calcular el área de este cuadrado, calcular L por L y X por X, el resultado de X por X y L por L lo sumo y se lo resto al resultado del grande", que es correcta, así como la estructura simbólica que expuso, al igual que E3. E3 estableció la estructura correctamente de forma verbal, "multiplicaríamos L por L a lo que nos dé y después lo que nos dé M por M, lo que nos dé creo que habría que sumarlo y se lo restamos a 36", pero no de forma simbólica. E5 estableció una relación que no es correcta, mientras que la de E6 sí.

También podemos comprobar el uso o no de dos símbolos diferentes para identificar cada una de las esquinas. Todos los estudiantes identificaron cada esquina con una letra diferente pero E2 e E4 no precisaron de ayuda por parte de la entrevistadora para ello. E3 inicialmente hizo uso de una sola letra al comentar "si no fueran iguales pues a uno le pondría L y a otro pues un número o una letra más grande, multiplicando L por L” y E6 hizo lo propio cuando dijo "las llamaría igual porque al fin y al cabo hago lo mismo, puedes utilizar la misma letra". 
Dada la presencia en esta ocasión de dos variables y la existencia de la condición $x+y=$ 6 que liga ambas en caso de rellenar todo el lado, analizamos también la respuesta de los estudiantes con base en este hecho. E1 estableció que la suma debe ser 36, con ayuda, pero no llegó a establecer ninguna estructura analítica. E2, también con ayuda, expuso que "juntos tienen que valer 6", expresando $L+x=6$. E3 no estableció la estructura pero E4 sí que lo hizo; asumió que los dos lados deben valer 6 entre los dos. E5 estableció la estructura de forma verbal y mediante sumas particulares pero no generalizó. E6 estableció la estructura $L+E=6$, que no siempre se cumple y, en caso de no hacerlo, ello implica que no se rellene todo el lado. Hace uso de representación verbal y simbólica.

En la tabla 12 presentamos ejemplos de las estructuras establecidas por los estudiantes.

Tabla 12.

Estructuras en la entrevista



Nota. - = no hay información disponible. 


\section{Representación}

Para la estructura en la entrevista planteamos dos tareas diferentes: se eliminan 4 esquinas iguales y se eliminan 2 esquinas opuestas diferentes. Con base en esto, se presentan los resultados obtenidos.

En la tabla 13 presentamos el resumen de la entrevista de los estudiantes en relación a la representación.

Tabla 13.

Representación en la entrevista

\begin{tabular}{lll}
\hline Estudiante & Tarea de las 4 esquinas & Tarea de las 2 esquinas \\
\hline E1 & Verbal & Simbólica \\
E2 & Verbal y pictórica & Verbal y pictórica \\
E3 & Verbal y simbólica & Verbal \\
E4 & Simbólica & Simbólica \\
E5 & Simbólica & Verbal y simbólica \\
E6 & Verbal y simbólica & Simbólica \\
\hline
\end{tabular}

En cuanto a la tarea relativa a las cuatro esquinas, cinco de los seis estudiantes establecieron una estructura particular de carácter verbal. E3, E4, E5 y E6 hicieron uso de una estructura simbólica; E2, E3 y E6 de tipo verbal y E2 también estableció la estructura de forma pictórica.

Prestando atención a la representación simbólica, E2 mencionó el uso de la potencia, pero lo hizo de forma errónea al notar $L^{8}$, debido a la presencia de ocho $L$. E3 también hizo uso de la potencia pero no agrupó bien los términos. Expuso que la estructura es " 6 por 6 36. Ahora L por L por L..., 36- $\mathrm{L}^{8}$ "; la expuso bien verbalmente pero no simbólicamente.

En relación a las tareas que implican dos esquinas diferentes, E1 hizo uso de una estructura simbólica. E2, E3 y E5 hicieron uso de la verbal; E4, E5 y E6 hicieron uso de la simbólica y E2 de la pictórica.

\subsection{Síntesis de resultados}

Presentamos una síntesis de resultados para cada una de las partes de la recogida de información. 


\section{Trabajo en el cuestionario}

Como síntesis de lo expuesto, podemos decir que, en cuanto a los valores extremos, los estudiantes no los establecieron de forma correcta salvo casos puntuales. En referencia al carácter de la variable, los estudiantes establecieron correctamente de forma general que la variable puede tomar valores decimales, pero no negativos. Uno de ellos no respondió, dos dan respuestas en el carácter decimal y uno en el negativo.

Con respecto al establecimiento de la estructura y la representación usada para ello, tres de los estudiantes la establecieron, aunque de forma errónea. Los tres hicieron uso de una representación verbal, usando uno de ellos además representación pictórica (representación múltiple).

\section{Puesta en común}

Atendiendo a la evolución de los estudiantes tras poner en común las preguntas, podemos decir que E2 finalmente estableció bien los valores de la variable y el valor máximo del área, estableciendo también E4 este último de forma correcta. De E1, E3 y E5 no se percibe evolución, dado que no interactuaron en la parte común y de E6 se obtiene la máxima evolución: en el trabajo individual dijo no ser posible determinar los valores por ser infinitos, determinando los cuatro correctamente en la puesta en común tras compartir los resultados con sus compañeros.

En cuanto al carácter decimal o negativo de las variables, se dispone de información relativa a tres estudiantes, siendo las respuestas de dos de los mismos correctas. El tercero falló en el carácter decimal de la variable, manteniendo el error cometido en el trabajo individual. E4 expuso que para poder tomar valores decimales, deben aparecer dos letras, la primera para la parte entera del número y la segunda, para la parte decimal.

En referencia al establecimiento de la estructura y la representación usada para ello, uno de los estudiantes la estableció sin necesidad de ayuda del investigador y de forma verbal. Otros cuatro estudiantes (pues el sexto no interactuó) cometieron errores en el establecimiento de la estructura y vieron necesario el uso de la regla para poder determinar el área. Sin embargo, los cinco establecieron la existencia de una estructura, la determinasen o no. 
El tipo de representación usado por los estudiantes es mayoritariamente verbal. Cinco estudiantes hicieron uso de este tipo de representación, tres de la simbólica y uno de la pictórica. La representación múltiple también se encuentra presente, siendo usada por tres estudiantes. Las combinaciones usadas en la representación múltiple son verbal-simbólica y verbal-simbólica-pictórica. Tras la ayuda por parte de los investigadores, los seis estudiantes llegaron a establecer la estructura, de forma verbal y simbólica.

\section{Entrevista}

Como síntesis de los resultados obtenidos durante la entrevista, podemos exponer que los estudiantes encontraron dificultades para establecer los valores extremos de ambas variables en ambas tareas. En la relativa a las 4 esquinas, tres fueron los estudiantes que respondieron bien al valor mínimo de la variable lado y otros tres, al valor máximo. El valor mínimo de la variable área fue establecido de forma correcta por tres estudiantes y el valor máximo, por cinco. Destacar que la ayuda por parte de los investigadores está presente siempre en el establecimiento de los valores extremos y el uso de intervalos en esta tarea por parte de algunos estudiantes (cuatro de ellos establecen intervalos, haciéndolo de forma correcta dos). En cuanto a la tarea de las 2 esquinas, tres estudiantes establecieron bien el valor mínimo de la variable lado, uno el valor mínimo de la variable área y dos su valor máximo. Al determinar el valor máximo de la variable lado los estudiantes presentaron dificultades por hacer uso de las áreas para establecerlo.

En relación al carácter de la variable lado, los estudiantes no presentaron dificultades para determinar que puede tomar valores decimales, pero no negativos. En la tarea de las 4 esquinas uno de los estudiantes no respondió al carácter decimal y en la de 2 esquinas, un estudiante no respondió al carácter entero y otro a ambos.

El tipo de representación usado por los estudiantes fue mayoritariamente simbólico y verbal. Cuatro estudiantes hicieron uso de la representación simbólica en ambas tareas, cuatro de la verbal en la tarea de las 4 esquinas y tres en la tarea de las 2 esquinas. La representación pictórica también se encuentra presente, siendo usada por un estudiante en ambas tareas (el mismo), así como la múltiple, usada por tres estudiantes en la tarea de las 4 esquinas y por dos en la de 2 esquinas. Las combinaciones usadas en la representación múltiple son verbal-simbólica y verbal-pictórica. 


\section{Capítulo 5. Conclusiones}

Estructuramos las conclusiones entorno a los siguientes apartados: (a) logro de objetivos de investigación, resultados significativos y comparación con estudios previos, (b) otros hallazgos, (c) limitaciones del estudio y (d) líneas abiertas de investigación para el futuro.

\section{Logro de objetivos de investigación}

La principal contribución del presente estudio estriba en la introducción de una tarea que implica variable continua y una función cuadrática en un contexto geométrico. Ello ha permitido evidenciar las capacidades de los estudiantes de generalizar en contextos funcionales diferentes a los usuales en la literatura, que suelen contemplar variable discreta y funciones lineales. Se da respuesta a los objetivos de investigación planteados para el presente estudio.

OE1. Describir el trabajo de los estudiantes sobre los valores de las variables implicadas en el problema.

En relación a los valores extremos que toman ambas variables, los estudiantes presentaron dificultades en el establecimiento de los mismos, no consiguiendo ninguno de los seis establecer los cuatro valores de forma correcta (aunque alguno de los estudiantes sí que obtuvo algún valor extremo correctamente). La ayuda proporcionada por los investigadores no permitió que establecieran bien dichos valores.

Consideramos que los estudiantes tomaron los valores extremos como casos extraños, como en el ejemplo mencionado anteriormente de los estudiantes que establecen como valores extremos 1 y 5. En otros trabajos (Cañadas y Fuentes, 2015; Pinto y Cañadas, 2018), los valores extremos de la variable no son preguntados a los estudiantes ni son finitos en los valores mínimo y máximo (suelen ser funciones con un mínimo finito, acotadas en el mínimo pero no en el máximo). En este caso, quizá los estudiantes no se lo han cuestionado porque no se les ha preguntado.

En cuanto al tipo de valores que puede tomar la variable independiente, los estudiantes presentaron algunas dificultades para determinar que el lado no puede tomar valores negativos pero sí decimales. Si bien es cierto que durante el trabajo individual en el cuestionario algunos cometieron errores o incluso no fueron capaces de dar una respuesta 
a las preguntas (E1), durante la entrevista todos aquellos estudiantes que respondieron lo hicieron de forma correcta. Cabe destacar que cuando se les pedía una justificación a sus respuestas, respondían de forma rápida que el carácter de la variable no puede ser negativo por no tener sentido una medida de longitud (lado de la esquina suprimida) negativa. Esto implica que los estudiantes fueron capaces de asumir que la letra o variable no solamente representa un número y puede ser sustituida por el mismo, sino que se asocia con una medida; en este caso, una longitud. Es en este hecho donde podemos comprobar que los estudiantes trabajaron de forma correcta con la variable continua. Hablaron de valores continuos en la variable cuando hicieron uso de los números decimales. Puede ser que ellos no supieran explícitamente de su trabajo con valores continuos; en cambio, de forma implícita sí lo hicieron porque asumieron que la variable lado puede tomar valores decimales, no solamente valores aislados. Así lo expusieron ellos mismos.

También es significativa una de las justificaciones de un estudiante cuando expuso que la variable lado no puede tomar valores decimales. Según este estudiante, para que la variable pudiera tomar valores decimales, debería constar de dos letras: una para la parte entera y otra para la decimal, explicación que puede proceder de los conocimientos previos de dicho estudiante en materia de número decimales (parte entera y decimal de los mismos).

OE2. Describir las estructuras establecidas por los estudiantes.

En cuanto a las estructuras, vemos evolución en las respuestas de los estudiantes. Inicialmente, son 3 los estudiantes que respondieron y ninguno de ellos expuso la estructura correcta (todos contestaron $36-L$, sin identificar la parte cuadrática). Los estudiantes llegaron a establecer $L \times L$ como área de la esquina eliminada, hecho que consideramos un logro importante por la generalización conseguida y la identificación de la estructura (equivalente a $L^{2}$ ). Sin embargo, cuando restaron esta cantidad a 36 (área del cuadrado original), no fueron capaces de agrupar como potencia cuadrada. Algunos estudiantes mencionaron la potencia, aunque no hicieron uso de ella de forma correcta. Ello puede deberse al hecho de que, si bien conocían la potencia aplicada a una situación con números, aritmética, no les era conocida en una situación con letras, algebraica (siendo el uso el mismo en ambas situaciones, pueda ser que los estudiantes no lo identifiquen como tal y consideren que el uso de la potencia es diferente con letras y con 
números). Puede deberse también al hecho de no haber adquirido bien los conocimientos previos relativos a la potencia.

En la tarea relativa a las 4 esquinas suprimidas (parte 4), tres de los cinco estudiantes que respondieron identificaron la estructura cuadrática correcta. En relación al hecho de usar la misma letra para las cuatro esquinas (pues son iguales las cuatro), cuatro de los cinco estudiantes lo tuvieron en cuenta y lo incluyeron en la estructura que establecieron. La dificultad de poner $4 L$ en lugar de $4 L^{2}$ puede provenir del hecho de que identificasen que debían quitarle cuatro veces algo que depende de $L$, pero no diferenciasen que $L$ fuese la longitud del lado o el área de la esquina suprimida.

En la tarea de las 2 esquinas (parte 5), vemos la estructura cuadrática (cuatro de cinco estudiantes la usaron) y la utilización de diferentes letras para designar cada esquina (cuatro de cinco estudiantes). Esto evidencia que los estudiantes asociaron que a esquinas diferentes le correspondían letras diferentes. También es interesante notar la agrupación de términos llevada a cabo por E4: en lugar de expresar $36-L^{2}-J^{2}$, hizo uso de otras letras para indicar los productos $(M \times M=N, L \times L=A)$, de forma que la relación final fue $36-N-A$.

En relación también a esta tarea, la presencia de dos variables y la existencia de la condición $x+y=6$ que liga ambas en caso de rellenar todo el lado, concluimos lo que sigue: el establecimiento de la relación entre $x$ e $y$ es una tarea que implicaba sentido espacial y sentido de la medida, pues algunos de los estudiantes pusieron ejemplos que eran imposibles por las medidas de $x$ e $y$. Por tanto, esta tarea es más compleja por intervenir tres variables (área, lado de un cuadrado, lado de otro...) y el hecho de que llegasen a abordarla los estudiantes es indicador de sus características del talento.

Evidenciamos que los estudiantes tienen capacidad para representar de forma verbal y simbólica. No rechazan la notación simbólica, pero cometen errores, por lo que no creemos que la dificultad provenga de la función cuadrática, sino por agrupación de términos o uso de la notación simbólica.

OE3. Identificar y describir los sistemas de representación usados por los estudiantes para expresar la estructura. 
De los cinco tipos de representación presentados en el marco conceptual (verbal, simbólica, tabular, pictórica y múltiple), los estudiantes hicieron uso de representaciones verbales, simbólicas, pictóricas y múltiples. La representación más usada fue el verbal. Esto puede deberse al uso del lenguaje común que requiere este tipo de representación, dado que, por el curso académico en el que se encuentran los estudiantes, aún no se les ha introducido el simbólico. A pesar de ello, esta representación es usada por los estudiantes. También encontramos la representación pictórica e incluso múltiple, como combinación de dos (verbal y pictórica; verbal y simbólica) o incluso tres (verbal, simbólica y pictórica) tipos de representación. Estos resultados son similares a lo encontrado por Cañadas y Fuentes (2015), que evidencian como representaciones más usadas la verbal y la simbólica, llegándose a combinar dos o tres tipos de representaciones diferentes.

La única representación que ningún estudiante utilizó es la tabular. Esto puede derivarse del poco uso que se hace de la misma en diferentes cursos, ya que las tablas se presentan mayoritariamente en contenidos relacionados con estadística.

Con base en la literatura, coincidimos con las conclusiones de Merino et al. (2013) en relación a la representación más usada: la verbal. En su caso, la mayoría de los estudiantes también hicieron uso de la representación pictórica y puntualmente, de la simbólica. Ante eso, nuestros resultados son diferentes: es mayor el número de estudiantes que hicieron uso de una representación simbólica para establecer la estructura, dado que un solo estudiante utiliza la representación pictórica (E2). De forma similar, nuestros resultados coinciden en materia de representación con los de Merino et al. (2013). Coincidimos en que quizá la elección de los estudiantes de la representación verbal es debida a la comodidad que implica para ellos en la exposición y justificación de sus respuestas.

\section{Otros hallazgos}

Relevante es también en este estudio el papel jugado por la ayuda por parte de los investigadores a los estudiantes durante las sesiones. Dichos estímulos ayudaron en varias ocasiones a los estudiantes a la consecución de las tareas, de forma similar a lo expuesto por Ureña et al. (2019), donde los investigadores evidencian que los estímulos influyeron mucho en las respuestas de los chicos. La reformulación de las preguntas, la verbalización de las reflexiones de los estudiantes o la repetición de las preguntas son algunos de los estímulos que se llevaron a cabo en las sesiones y, de acuerdo con Hidalgo y Cañadas 
(2020), permitieron a los estudiantes en diversas ocasiones cambiar la estrategia en la que se aproximaban a la tarea o comprender lo que se les estaba pidiendo en la misma.

\section{Limitaciones del estudio}

En cuanto a las limitaciones del estudio, los estudiantes participantes pertenecen todos al mismo centro. Esto implica la presencia de características muy similares entre sí. Aunque dicha característica ha facilitado la realización de las entrevistas, sería interesante ampliar a otros centros.

Si bien han sido varias las dificultades matemáticas discriminadas por el hecho de ser estudiantes parte de un programa de enriquecimiento curricular (buena actitud hacia las tareas matemáticas, presencia de conocimientos previos), comprendemos que los resultados obtenidos vienen sesgados por la propia muestra. Aun así, reconocemos que los estudiantes han presentado dificultades y errores en el proceso de resolución de la tarea, lo que aporta información muy relevante para las dificultades que podrían derivarse en los estudiantes ordinarios.

El presente trabajo contempla dos características nuevas asociadas a la relación funcional que implica la tarea: la estructura cuadrática y la variable continua. La introducción de ambas no nos ha permitido profundizar en cada una de forma independiente y obtener conclusiones más precisas en cada una de ellas.

\section{Líneas abiertas de investigación para el futuro}

Son varias las líneas o preguntas de investigación que quedan abiertas para el futuro. El presente trabajo introduce dos características nuevas asociadas a la relación funcional que implica la tarea: la estructura cuadrática y la variable continua. Este hecho genera dos preguntas: en primer lugar, la implementación de una tarea de variable discreta en un grupo de estudiantes que formen parte de programas de enriquecimiento curricular. En segundo lugar, la implementación de una tarea de variable continua (que podría ser la misma del presente trabajo) en un grupo de estudiantes que no formen parte de programas de enriquecimiento curricular. En consecuencia, podrían llevarse a cabo investigaciones que indaguen en cada una de las características de forma aislada: función lineal de variable continua o función cuadrática de variable discreta. Podría hacerse primero con 
niños en programas de enriquecimiento curricular, analizar si las dificultades son mayores o no que los resultados presentados en nuestro trabajo y a continuación, contemplar llevarlos a cabo en etapas educativas anteriores.

Hay estudios que justifican el desarrollo del pensamiento funcional en edades tempranas como pueden ser primeros cursos de educación primaria (Cañadas, Castro y Castro, 2008; Cañadas y Fuentes, 2015) o incluso educación infantil (Blanton y Kaput, 2004). Existe carencia en la literatura de estudios que impliquen variable continua en dichas etapas educativas, por lo queesta línea de investigación queda también abierta. 


\section{Referencias}

Asencio, M. J., Romero, J. A. y de Vicente, E. (2003). Estadística. Madrid, España: McGraw-Hill Interamericana de España S.L.

Ayala-Altamirano, C. y Molina, M. (2019). Meanings attributed to letters in functional contexts by primary school students. International Journal of Science and Mathematics Education. https://doi.org/10.1007/s10763-019-10012-5

Benavides, M., Maz, A., Castro, E. y Blanco, R. (2004). La educación de niños con talento en Iberoamérica. Santiago de Chile: Oficina Regional de Educación de la Unesco para América Latina y el Caribe.

Blanton, M. y Kaput, J. (2004). Elementary grades students' capacity for functional thinking. En M. Johnsen y A. Berit (Eds.), Proceedings of the 28th International Group of the Psicology of Mathematics Education (Vol. 2, pp. 135-142). Bergen, Noruega: Bergen University College.

Blanton, M. y Kaput, J. (2011). Functional thinking as a route into algebra in the elementary grades. En J. Cai y E. Knuth (Eds.), Early algebraization (pp.5-23). Berlín, Alemania: Springer.

Blanton, M., Levi, L., Crites, T. y Dougherty, B. (2011). Developing essential understanding of algebraic thinking for teaching mathematics in Grades 3-5. Reston, VA: NCTM.

Blanton, M., Brizuela, B., Gardiner, A., Sawrey, K. y Newman-Owens, A. (2015). A learning trajectory in 6-year-olds'thinking about generalizing functional relationships. Journal for Research in Mathematics Education, 46(5), 511-558.

Brizuela, B. y Blanton, M. (2014). El desarrollo del pensamiento algebraico en niños de escolaridad primaria. Revista de Psicología - Segunda épica (UNLP), 14, 37-57.

Cañadas, M. C., Brizuela, B. y Blanton, M (2016). Second graders articulating ideas about linear functional relationships. The Journal of Mathematical Behavior, 41, 87103. http://doi.org/10.1016/j.jmathb.2015.10.004

Cañadas, M. C., Castro, E. y Castro, E. (2008). Patrones, generalización y estrategias inductivas de estudiantes de $3^{\circ}$ y $4^{\circ}$ de Educación Secundaria Obligatoria en el problema de las baldosas. PNA, 2(3), 137-151.

Cañadas, M. C. y Figueiras, L. (2011). Uso de representaciones y generalización de la regla del producto. Infancia y aprendizaje, 34(4), 409-425.

Cañadas, M. C. y Fuentes, S. (2015). Pensamiento funcional de estudianes de primero de educación primaria: un estudio exploratorio. En C. Fernández, M. Molina y N. Planas (Eds.), Investigación en Educación Matemática XIX (pp. 211-220). Alicante, España: SEIEM.

Cañadas, M. C. y Molina, M. (2016). Una aproximación al marco conceptual y principales antecedentes del pensamiento funcional en las primeras edades. En E. Castro, E. Castro, J. L. Lupiáñez, J. F. Ruiz y M. Torralbo (Eds.), Investigación en Educación Matemática. Homenaje a Luis Rico (pp.209-218). Granada, España: Comares.

Castro, E., Cañadas, M. C. y Molina, M. (2017). Pensamiento funcional mostrado por estudiantes de Educación Infantil. Edma 0-6: Educación Matemática en la Infancia, 6(2), 1-13. 
Common Core State Standards Initiative (CCSI 2010). Common Core State Standards for Mathematics. Washington, DC: National Governors Association Center for Best Practices and the Council of Chief State School Officers.

Confrey, J. y Smith, E. (1991). A framework for functions: Prototypes, multiple representations, and transformations. En R. G. Underhill (Ed.), Proceedings of the 13th anual meeting North American Chapter of the International Group for the Psychology of Mathematics Education (Vol. 1, pp. 57-63). Blacksburg, VA: Conference Committee.

Doorman, M. y Drijvers, P. (2011). Algebra in function. En P. Drijvers (Ed.), Secondary algebra education: Revisiting topics and themes and exploring the unknown (119135). Rotterdam, Países Bajos: Sense Publishers.

Drijvers, P. (2003). Learning algebra in a computer algebra environment: Design research on the understanding of the concept of parameter. Tesis doctoral no publicada. Utrecht, Los Países Bajos: Utrecht University.

Drijvers, P., Dekker, T. y Wijers, M. (2011). Patterns and formulas. En P. Drijvers (Ed.), Secondary algebra education (pp. 89-100). Rotterdam, Países Bajos: Sense Publishers.

Freiman, V. (2006). Problems to discover and to boost mathematical talent in early grades: A challenging situations approach. The Montana Mathematics Enthusiast, 3 (1), 51-75. Canadá: The Montana Council of Teachers of Mathematics.

Freudenthal, H. (1983). Didactical phenomenology of mathematical structures. Dordrecht, Países Bajos: Reidel.

Friedlander, A. y Tabach, M. (2001). Promoting multiple representations in algebra. The roles of representation in school mathematics, 173-185.

Goldin, G. y Shteingold, N. (2001). Systems of representations and the development of mathematical concepts. En A. Cuoco y F. Curcio (Eds.), Roles of representations in school mathematics - 2001 Yearbook (pp. 1-23). Reston, VA: National Council of Teachers of Mathematics.

Greenes, C. (1981). Identifying the gifted student in mathematics. Arithmetic Teacher, 28 (8), 14-17.

Heid, M. K. (1996). A technology-intensive functional approach to the emergence of algebraic thinking. En A. Bednarz, C. Kieran y L. Lee (Eds.), Approaches to algebra: Perspectives for research and teaching (pp. 239-255). Dordrecht, Países Bajos: Kluwer Academic Publishers.

Henández, R., Fernández, C. y Baptista, P (2010). Metodología de la investigación (5 ed.). México, DF: McGraw-Hill.

Hidalgo-Moncada, D. y Cañadas, M. C. (2020). Intervenciones en el trabajo con una tarea de generalización que involucra las formas directa e inversa de una función en sexto de primaria. PNA, 14(3), 204-225.

Kaput, J., Carraher, D. W. y Blanton, M. L. (2008). Algebra in the early grades. Londres, Reino Unido: Routlege.

Kaput, J. (1999). Teaching and learning a new algebra. En E. Fennema y T. A. Romberg (Eds.), Mathematics classrooms that promote understanding (pp. 133-155). Mahwah, NJ: Lawrence Erlbaum Associates

Kaput, J. (2000). Transforming algebra from an engine of inequity to an engine of mathematical power by "algebrafying" the K-12 curriculum. Dartmouth, MA: National Center for Improving Student Learning and Achievement in Mathematics and Science. 
Kieran, C. (1989). The early learning of algebra: A structural perspective. En S. Wagner y C. Kieran (Eds.), Research issues in the learning and teaching of algebra, 4, 3356. Reston, VA: NCTM.

Kolloffel, B., Eysink, T. H., de Jong, T. y Wilhelm, P. (2009). The effects of representational format on learning combinatorics from an interactive computer simulation. Instructional Science, 37(6), 503-517.

Krutetskii, V. A. (1976). The psychology of mathematical abilities in school children. Chicago: University of Chicago Press.

Lloyd, G., Beckmann, S., Zbiek, R. M. y Cooney, T. (2010). Developing essential understanding of functions for teaching mathematics in Grades 9-12. Resto, VA: National Council of Teachers of Mathematics.

Mason, J., Stephens, M. y Watson, A. (2009). Appreciating mathematical structure for all. Mathematics Education Research Journal, 21(2), 10-32.

Merino, E. (2012). Patrones y representaciones de alumnos de $5^{\circ}$ de educación primaria en una tarea de generalización. Trabajo Fin de Máster, Universidad de Granada. Disponible en http://funes.uniandes.edu.co/1926/

Merino, E., Cañadas, M. C. y Molina, M. (2013). Uso de representaciones y patrones por alumnos de quinto de educación primaria en una tarea de generalización. Edma O6: Educación Matemática en la Infancia, 2(1), 24-40.

Ministerio de Educación y Ciencia (2014). Real Decreto 126/2014 de 28 de febrero, por el que se establece el currículo básico de la Educación Primaria. BOE, 52, 19.34919.420).

Molina, M. (2005). La integración del pensamiento algebraico en Educación Primaria. En M. Camacho, P. Flores y M. P. Bolea (Eds.), Investigación en Educación Matemática XI (pp. 53-69). San Cristóbal de la Laguna, España: SEIEM.

Molina, M. (2015). Concepciones del álgebra escolar. Granada: Dpto. Didáctica de la Matemática, Universidad de Granada.

Molina, M. y Cañadas, M. C. (2018). La noción de estructura en el early algebra. En P. Flores, J. L. Lupiáñez e I. Segovia (Eds.), Enseñar matemáticas. Homenaje a los profesores Francisco Fernández y Francisco Ruiz (pp. 129-141). Granada, España: Atrio.

Morales, R. A., Cañadas, M. C., Brizuela, B. M. y Gómez, P. (2018). Relaciones funcionales y estrategias de alumnos de primero de Educación Primaria en un contexto funcional. Enseñanza de las Ciencias, 36(3), 59-78.

Moss, J., Beatty, R., Shillolo, G. y Barkin, S. (2008). What is your theory? What is your rule? Fourth graders build their understanding of patterns and functions on a collaborative database. En C. Greenes (Ed.), Algebra and algebraic thinking in school mathematics: The National Council of Teachers of Mathematics $70^{\text {th }}$ Yearbook (2008) (pp. 155-168). Reston, VA: NCTM.

Mulligan, J., English, L. D., Mitchelmore, M. y Robertson, G. (2010). Implementing a pattern and structure mathematics awareness program (PASMAP) in Kindergarten. En L. Sparrow, B. Kissane y C. Hurst (Eds.), Shaping the future of mathematics education: Proceedings of the 33rd annual conference of the Mathematics Education Research Group of Australasia (pp. 795-802). Fremantle, Australia: MERGA.

National Council of Teachers of Mathematics (NCTM). Principles and Standars for School Mathermatics. Reston, VA: NCTM, 2000. 
Pinto, E. y Cañadas, M. C. (2018). Generalization in fifth graders within a functional approach. PNA. Revista de Investigación en Didáctica de la Matemática, 12(3), 173-184.

Pinto, E. y Cañadas, M. C. (2019). Generalizations of third and fifth graders within a functional approach to early algebra. Mathematics Education Research Journal. DOI: 10.1007/s13394-019-00300-2

Pinto, E., Cañadas, M. C., Moreno, A. y Castro, E. (2016). Relaciones funcionales que evidencian estudiantes de tercero de educación primaria y sistemas de representación que usan. En J. A. Macías, J. L. González, M. T. Sánchez, P. Hernández, C. Fernández, F. J. Ruiz, T. Fernández y A. Berciano (Eds.), Investigación en Educación Matemática XX (pp. 417-426). Málaga, España: SEIEM.

Radford, L. (1998). On signs and representations. A cultural account, Scientia Paedagogica Experimentalis, 35(1), 277-302.

Ramírez, R. (2012). Habilidades de visualización de los alumnos con talento matemático (Tesis doctoral). Universidad de Granada, Granada, España.

Rico, L. (2007). La competencia matemática en PISA. PNA, 1(2), 47-66.

Rico, L. (2009). Sobre las nociones de representación y comprensión en la investigación en educación matemática. PNA, 4(1), 1-14.

Schliemann, A. D., Carraher, D. W. y Brizuela, B. M. (2012). Algebra in elementary school. En L. Coulangey y J.-P. Drouhard (Eds.), Enseignement de l'algèbre élémentaire: Bilan et perspective (pp. 109-124). (Número especial de Recherches en Didactique des Mathématiques)

Smith, E. (2008). Representational thinking as a framework for introducing funcions in the elementary curriculum. En J. J. Kaput, D. W. Carraher y M. L. Blanton (Eds.), Algebra in the early grades (pp. 133-160). Nueva York, NY: Routledge.

Torres, M. D., Cañadas, M. C. y Moreno, A. (2019). Estructuras y representaciones de alumnos de $2^{\circ}$ de primaria en una aproximación funcional del pensamiento algebraico. En J. M. Marbán, M. Arce, A. Maroto, J. M. Muñoz-Escolano y A. Alsina (Eds.), Investigación en Educación Matemática XXIII (pp. 573-582). Valladolid, España: SEIEM.

Ureña, J., Ramírez, R. y Molina, M. (2019). Representations of the generalization of a functional relationship and the relation with interviewer's mediation. Infancia $y$ Aprendizaje, 42(3), 570-614.

Usiskin, Z. (1988). Conceptions of school algebra and uses of variables. En A. Coxford (Ed.), The ideas of algebra K-12 (pp. 8-19). Reston, VA: National Council of Teachers of Mathematics.

Usiskin, Z., Peressini, A. L., Marchisotto, E. and Stanley, D. (2003). Mathematics for High School Teachers: An Advanced Perspective. Upper Saddle River, N.J.: Pearson Education.

Van Someren, M. W., Reimann, P., Boshuizen, H. P. A. y de Jong, T. (Eds.) (1998). Learning with multiple representations. Amsterdam, Países Bajos: Pergamon.

Warren, E. y Cooper, T. J. (2005). Introducing functional thinking in Year 2: A case study of early algebra teaching. Contemporary Issues in Early Childhood, 6(2), 150162.

Warren, E., Miller, J. y Cooper, T. J. (2013). Exploring young students' functional thinking. PNA, 7(2), 75-84.

Wilkie, K. J. (2016). Students' use of variables and multiple representations in generalizing functional relationships prior to secondary school. Educational Studies in Mathematics, 93(3), 333-361. 